\title{
Characterisation of uniform momentum zones in adverse pressure gradient turbulent boundary layers
}

\author{
A. Thavamani ${ }^{1}$, C. Cuvier $^{2}$, C. Willert ${ }^{3}$, J. M. Foucaut ${ }^{2}$, C. Atkinson ${ }^{1}$, and J. \\ Soria $^{1}$ \\ ${ }^{1}$ Laboratory for Turbulence Research in Aerospace and Combustion, Department of Mechanical and \\ Aerospace Engineering, Monash University, Melbourne, Australia \\ ${ }^{2}$ Ecole Centrale Lille, F-59650 Villeneuve d'Ascq, France \\ ${ }^{3}$ German Aerospace Center (DLR), Institute of Propulsion Technology, Köln, Germany
}

December 21, 2019

\begin{abstract}
Uniform momentum zones (UMZs) for an adverse pressure gradient turbulent boundary layer (APG-TBL) are characterised in this paper. The database described by Cuvier et al. [2017], which was obtained using 2-component 2-dimensional (2C-2D) particle image velocimetry (PIV) at two free-stream velocities of $5 \mathrm{~m} / \mathrm{s}$ and $9 \mathrm{~m} / \mathrm{s}$, is used for this study. The methodology described by Adrian et al. [2000] is used to identify and hence, statistically characterise the UMZs and UMZ interfaces in the APG-TBL. This study shows that the number of detected UMZs increases in the streamwise direction for both free-stream velocities. This increase is primarily thought to be due to the adverse pressure gradient (APG) since a comparison of the results between the two free-stream velocity APG-TBLs did not show any statistically significant difference with $R e_{\theta}$ for the range of Reynolds number examined in this study. The thickness of the UMZs, $\delta_{w}$ and the jump in streamwise velocity across the UMZ interface were found to increase in the wall-normal direction, whereas the width of the UMZ interface, $\Delta_{u}$, was found to decrease. The number of UMZs, $N_{u m z}$, identified appeared to influence the modal velocities/uniform velocities, geometrical properties of the UMZs and statistics of the flow. With increasing $N_{u m z}$, the modal velocities of the UMZs moved to higher values to accommodate new UMZs that developed closer to the wall and the thickness of each UMZ and $\Delta_{u}$ across the UMZ interface decreased, whereas $\delta_{w}$ increased. A slight difference in the magnitude of streamwise Reynolds stress and Reynolds shear stress was observed in the inner and outer regions, and a slight difference in the magnitude of turbulence production was also observed in the inner region between the velocity fields having a low or high number of UMZs indicating that the flow statistics are influenced by the number of UMZs present in the APG-TBL.
\end{abstract}

\section{Introduction}

Over several decades, the structural organisation of turbulent boundary layers (TBLs) has been the major focus of numerous investigations with a number of them reporting that TBLs 
are populated by a hierarchy of organised coherent structures [Theodersen, 1952, Kline et al., 1967, Wallace et al., 1972, Offen and Kline, 1974, Blackwelder and Eckelmann, 1979, Head and Bandyopadhyay, 1981, Adrian et al., 2000, Marusic, 2001]. Included in these structures are low and high-speed streaks $\left(\mathrm{y}^{+}>10\right)$, ejections, sweeps, streamwise vortices, spanwise vortices and hairpin vortices, as well as large-scale motions (bulges, super layers, deep valleys) in the outer layer. These coherent structures do not feature in isolation, but are spatially and temporally related. Investigations on the coherent structures have been carried out at low, moderate and high Reynolds number to understand their structural organisation, their scaling and their role in production and dissipation of turbulence. Kline et al. [1967] showed that the low speed streaks are formed close to the wall and undergo a process of interaction which includes lift up, an oscillation, a bursting and an ejection, suggesting an instability mechanism which plays a pivotal role in the transport of kinetic energy.

Wallace et al. [1972] describes ejections and sweeps as near wall $\left(5<y^{+}<35\right)$ motions moving away from or towards the wall with ejections corresponding to the movement of the flow away from the wall due to negative streamwise fluctuations $\left(u^{\prime}<0\right)$ and positive wall-normal fluctuations $\left(v^{\prime}>0\right)$ referred to as Q2 events, while sweeps correspond to the movement of flow towards the wall due to positive streamwise fluctuations $\left(u^{\prime}>0\right)$ and the negative wall-normal fluctuations $\left(v^{\prime}<0\right)$ referred to as Q4 events. These structures contribute to the majority of turbulence production $\left(-u^{\prime} v^{\prime} \frac{d U}{d y}\right)$ in the near-wall region of wall-bounded flow due to the anticorrelation of $u^{\prime}, v^{\prime}$ and a strong mean shear surrounding them [Wallace et al., 1972]. Following this, Offen and Kline [1975] associated the lift up of low speed streaks with hairpin vortex structures. The idea of the hairpin vortex was introduced by Theodersen [1952], who suggested that these vortices are responsible for turbulence transport and grow outward with the head of the vortices inclined at an angle of approximately 45 degrees to the wall. Hairpin vortices are formed in the near wall region of low momentum, with the leg of the vortices located near the wall and extending to the head of the vortices away from the wall. The legs of the hairpin vortices are often associated with streamwise vortices [Adrian, 2007], while the head is associated with spanwise vortices.

Experimental support for the existence of hairpin vortices in the outer layer at high Reynolds number was provided by the flow visualisations of Head and Bandyopadhyay [1981], who showed that hairpin vortices or vortex loops are commonly present in the TBL, originating from the near-wall region and inclined at an approximate angle of 45 degrees to the wall. They also reported the variations in the shape of these vortices from low to high Reynolds number and its scaling in the spanwise direction, including the development of these structures from loops to horseshoes to hairpin vortices at high Reynolds number, also showing that the aspect ratio and size of these vortices scale with Reynolds number. A further important observation by Head and Bandyopadhyay [1981] is the clustering of these vortices to form a larger coherent cluster of structures at higher Reynolds number, suggesting that these large coherent structures occurred consistently along the TBL as a series of hairpin vortices, indicating that they develop from the wall at regular intervals. These early findings were supported by Acarlar and Smith [1987b], who observed that the inclusion of a single hairpin in the laminar boundary layer led to the development of a number of hairpins which cluster together to form a large coherent structure travelling at an approximately constant velocity.

Adrian et al. [2000] quantitatively observed these coherent cluster of vortices in zero pressure gradient turbulent boundary layers (ZPG-TBLs) at moderate Reynolds numbers ranging from $R e_{\tau}=350$ to 2,000 using PIV measurements in the streamwise-wall-normal plane. They 
hypothesised that these vortices are associated with the heads of individual hairpin vortices that join together, forming a larger vortex packet that convects at a uniform velocity, with an approximate streamwise extent of 1.5 boundary layer thicknesses at an inclination angle to the wall varying between 3 - 35 degrees. Adrian et al. [2000] suggested that these larger vortex packets in the outer region propagate at a faster velocity compared to the smaller packets in the inner wall region, with the larger packets tending to form bulges as they reached the boundary layer thickness. Based on these observations Adrian et al. [2000] constructed a conceptual model which illustrates the observed features of the low-speed fluid regions formed by the coherent alignment of vortices that are much longer than the single hairpin vortices. Subsequently, Marusic [2001] showed that using a cluster of hairpin vortices provides greater agreement of model results with experimental data compared to results of models that are only based on individual hairpin vortices.

Subsequent 2-component 2-dimensional (2C-2D) PIV studies of velocity fields in the streamwisewall-normal and spanwise planes of a ZPG-TBL ranging from the buffer layer to the log region by Tomkins and Adrian [2003] reported that the ZPG-TBL was populated by low momentum regions of fluid elongated in the streamwise direction and bounded/bordered by hairpin vortices. This observation further supports the hairpin packet model hypothesised by Adrian et al. [2000]. Ganapathisubramani et al. [2003] investigated the hairpin packets of a ZPG-TBL at $R e_{\theta}$ $=2,500$, with $\theta$ denoting the boundary layer thickness, using spanwise 2C-2D PIV, showing that hairpin packets contribute a large percentage of the Reynolds shear stresses $(25 \%)$ in the log region although they only occupy a small area in the ZPG-TBL (4\% of total area), serving as an integral part of turbulence transport. A further study by Ganapathisubramani et al. [2005] showed that these vortices are inclined at an angle of approximately 45 degrees to the wall which is consistent with the observation of Head and Bandyopadhyay [1981], as well as the more recent observations of Hutchins et al. [2005]. Recently, Lee and Sung [2011] using DNS of ZPG-TBL at $R e_{\theta}=570-2,560$ showed that hairpin vortices are coherently aligned in the streamwise direction and create a streamwise region of low and high momentum with extent in the order of 3-4 boundary layer thicknesses.

A notable feature of ZPG-TBLs first reported by Meinhart and Adrian [1995] are regions of approximately uniform momentum. Meinhart and Adrian [1995] measured the instantaneous 2C-2D velocity field in the streamwise-wall-normal plane of a ZPG-TBL using PIV and observed from their instantaneous $2 \mathrm{C}-2 \mathrm{D}$ velocity field measurements that there were regions within the ZPG-TBL with approximately uniform streamwise momentum, which they named uniform momentum zones (UMZs). They found that these UMZs are commonly present in the logarithmic region of ZPG-TBL and that the wall-normal extent of these zones is proportional to the wall-normal distance, i.e. the thickness of UMZs closer to the wall is always smaller than the thickness of UMZs in the log and outer regions. They also observed thin regions of large wall-normal streamwise velocity gradients, i.e. $d u / d y$, across the layer separating the UMZs.

In a more extended study of UMZs in ZPG-TBL Adrian et al. [2000] observed the presence of UMZs in ZPG-TBL with streamwise extent of the order of $\delta$. They suggested that the origin of UMZs is the clustering of low momentum regions under the head of multiple hairpin vortices that are organised coherently in the streamwise direction. Adrian et al. [2000] used the probability density function (p.d.f.) of the instantaneous streamwise velocity component to identify the presence of UMZs in the ZPG-TBL in their study. Within their range of Reynolds number investigated, i.e. $R e_{\tau}=350-2,000$, no increase in the number of UMZs was observed. Recently, the UMZ in ZPG-TBL over a wider range of Reynolds numbers, $R e_{\tau}=1,200-14,500$, 
were investigated by de Silva et al. [2016] using the methodology proposed by Adrian et al. [2000]. They found that the number of UMZs increases approximately log-linear with Reynolds number. The geometrical properties of UMZ, such as the thickness of the UMZs, the jump in streamwise velocity across the UMZ interface and the width of the UMZ interface were investigated. Their results show that the thickness of UMZs, $\delta_{w}$, increases with wall-normal distance, whereas the jump in streamwise velocity across the UMZ interface decreases with wall-normal distance. They also reported that the width of the UMZ interface, $\Delta_{u}$, increases with wall-normal distance. In the present study of UMZs and UMZ interfaces in APG-TBLs, the methodology proposed by Adrian et al. [2000] is also used.

Overall, much progress has been made to understand the organisation of coherent structures in the TBL. The effort of this work to-date has been focused in characterising UMZs and understanding these characteristics in the ZPG-TBLs. However, the UMZs in a TBL subjected to an adverse pressure gradient have not been characterised, although APG-TBL flows are more commonly encountered in engineering applications such as turbine blades, diffusers, aerofoils etc than ZPG-TBLs. This paper reports on the first such characterisation of UMZ in high Reynolds number APG-TBLs, where once the UMZs are identified, their prevalence is investigated as a function of streamwise flow direction in the APG-TBL, as well as their geometric properties, UMZ interface characteristics and conditional turbulence statistics.

\section{Experimental database for the APG-TBL}

The APG-TBL database used for the present study emanated from an APG-TBL experiments, which were conducted in the LMFL Boundary Layer Windtunnel. This facility has a test section length of $20.6 \mathrm{~m}$, is $2 \mathrm{~m}$ wide and $1 \mathrm{~m}$ high. An extensive measurement campaign, which is fully documented in Cuvier et al. [2017], was undertaken in the framework of the EuHIT European project (www.euhit.org). Two free-stream velocities were employed for these experiments, namely $U_{\infty}=5 \mathrm{~m} / \mathrm{s}$ and $U_{\infty}=9 \mathrm{~m} / \mathrm{s}$, which are the reference free-stream velocities in the ZPG domain located upstream of the APG domain. The LMFL Boundary Layer Windtunnel with the model used to generate the APG-TBL is shown in in figure 1. The full characterisation of the experimental flow environment, including pertinent characteristics such as the streamwise static pressure coefficient distribution (i.e. figure 12 in Cuvier et al. [2017] ), definitions and distributions of pertinent quantities such as boundary layer thickness $\delta$ and free-stream velocity distributions $U_{e}$, as well as full details of the PIV measurement methodologies, and PIV data post-processing is given in Cuvier et al. [2017] and will not be repeated here. However, pertinent details of the 2C-2D PIV experiment are briefly presented with the more complete description provided in Cuvier et al. [2017].

For the $2 \mathrm{C}-2 \mathrm{D}$ PIV experiments, seeding particles with a mean diameter, $d_{p} \approx 1 \mu \mathrm{m}$, were introduced into the tunnel using a PEG smoke generator. The single-exposed PIV image pair acquisition was undertaken using 16 synchronised sCMOS cameras, which imaged the entire 3.5 $m$ streamwise extent of the APG region. This permitted the entire APG-TBL instantaneous $2 \mathrm{C}-2 \mathrm{D}$ velocity field along the centreline of the windtunnel to be measured. The field of view for each camera was approximately $230 \mathrm{~mm}$ in the streamwise direction and $255 \mathrm{~mm}$ in the wall-normal direction. A calibration target which extended the entire APG-TBL region was used to calibrate the location of each camera image in the APG-TBL domain. Details of the calibration and the stitching together of the individual velocity fields into one global velocity field are given in Cuvier et al. [2017]. 
Table 1: Main parameters of streamwise-wall-normal 2C-2D PIV measurements [Cuvier et al., 2017].

\begin{tabular}{ll}
\hline Parameters & \\
\hline Field of View $(\mathrm{m} \times \mathrm{m})$ & $3.466 \times 0.255$ \\
Final IW size (px x px) & $24 \times 24$ \\
IW overlap & $75 \%$ \\
Vector field & $3,250 \times 238$ \\
Number of velocity vector fields samples & 30,000 \\
Frequency $(\mathrm{Hz})$ & 4 \\
Seeding particle size $(\mu \mathrm{m})$ & 1 \\
\hline
\end{tabular}

A light sheet $300 \mathrm{~mm}$ in height at the beginning of the field of view and $1 \mathrm{~mm}$ in thickness all along the $3.5 \mathrm{~m}$ of the APG region was generated using an Nd:YAG laser and appropriate laser shaping optics. This constant thickness was obtained by using a spherical lens with a focal length of $7.5 \mathrm{~m}$ with the laser introduced downstream of the APG ramp via a mirror placed in the free stream. The light sheet was carefully set tangent to the ramp wall of the APG region to minimise light reflection from it. With this set-up, the whole field of view of the 16 sCMOS camera used in the experiment (i.e. 3,500 $\mathrm{mm} \times 250 \mathrm{~mm}$ ) was covered with sufficient illumination.

The single-exposed PIV image pairs were captured using the 16 sCMOS cameras with 30,000 image pairs recorded at the frequency of $4 \mathrm{~Hz}$ for both free-stream velocities of $5 \mathrm{~m} / \mathrm{s}$ and $9 \mathrm{~m} / \mathrm{s}$. The 2C-2D velocity field was determined using digital cross-correlation PIV analysis Willert and Gharib [1991] using a multigrid/multipass digital cross-correlation PIV algorithm [Soria, 1996, 1998]. Four multigrid/multipass passes were performed with interrogation window (IW) size starting with $64 \times 64$ down to $24 \times 24$. To further improve the results image deformation was carried out prior to the last pass with a cubic spline interpolation of the grey level and bilinear interpolation for the displacement. Also, to reduce the effect of laser reflection and camera noise background subtraction was undertaken prior to multigrid/multipass digital cross-correlation PIV analysis.

The pertinent experimental parameters are summarised in Table 1 . The pertinent APGTBL parameters for the free-stream velocity of $5 \mathrm{~m} / \mathrm{s}$ and $9 \mathrm{~m} / \mathrm{s}$ are given in Table 3 and 4 respectively of Cuvier et al. [2017]. The resulting instantaneous velocity fields have 3,250 vectors along the streamwise direction and 238 in the wall-normal direction. The instantaneous velocity components in the streamwise and wall-normal directions are denoted by $u$ and $v$ respectively with the associated fluctuating velocity components by $u^{\prime}$ and $v^{\prime}$, which are obtained by subtracting the mean velocity field from the instantaneous velocity field, i.e. $u^{\prime}=u-U$ and $v^{\prime}=v-V$. The mean velocity components are denoted by $U, V$, obtained by ensemble averaging of $u$ and $v$ over the 30,000 2C-2D PIV velocity field samples. The boundary layer parameters used to characterise the APG region, their definitions and method of calcualation are provided in Appendix A.1.

\section{Detection of Uniform Momentum Zones}

A UMZ represents a region of the flow with an approximately uniform streamwise momentum (i.e. the streamwise velocity, which is the specific streamwise momentum) as defined by 

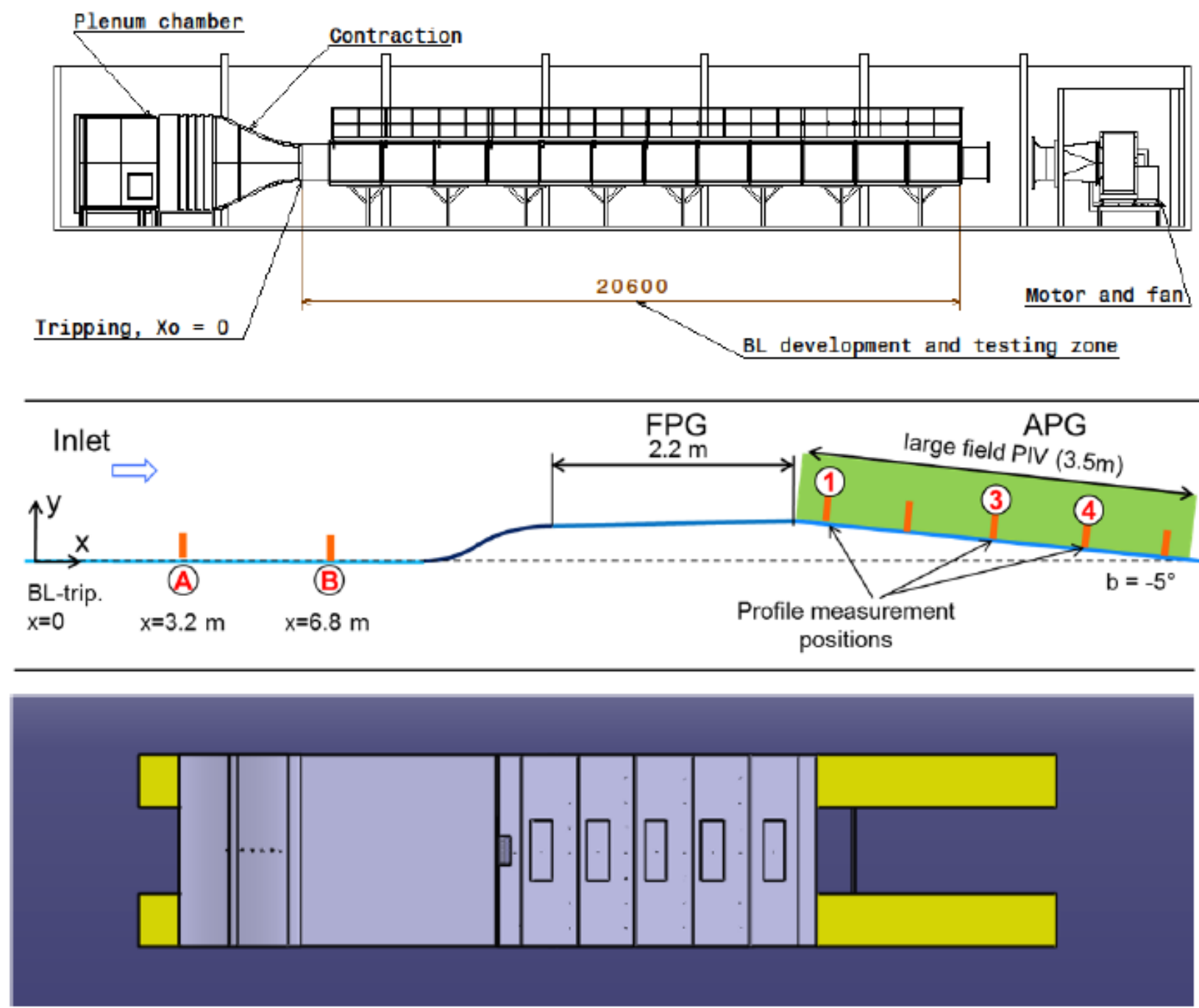

Figure 1: (top) Sketch of LML wind tunnel(front view),(middle) Schematic representation of the wind tunnel test section, (bottom) Top view of the experimental platform showing the position of the windows [Cuvier et al., 2017].

[Meinhart and Adrian, 1995]. This approximate uniformity of the streamwise velocity in these regions leads to local maxima, i.e. peaks, in the p.d.f. of the streamwise velocity component. The corresponding velocities of these peaks are referred to as modal velocity, i.e. the uniform velocity of each region. The number of modal velocities detected equates to the number of UMZs, which is denoted by $N_{u m z}$ and quantifies the number of zones present in a TBL [Adrian et al., 2000]. However, the instantaneous velocity fields obtained by measuring a TBL includes regions of turbulent and non-turbulent flow. The inclusion of non-turbulent flow in the construction of a p.d.f. of streamwise velocity can affect the detection of UMZs. In the recent study of UMZs in a ZPG-TBL, de Silva et al. [2016] reported that the omission of velocity vectors in the non-turbulent regions is necessary for the sensible detection of UMZs. Hence, in the present study, the interface between turbulent and non-turbulent regions (TNTI) in the APG-TBL is determined using the local variance of velocity fluctuations (local turbulent kinetic energy) as proposed by [Chauhan et al., 2014] (refer to Appendix A.2 for details). After the identification 


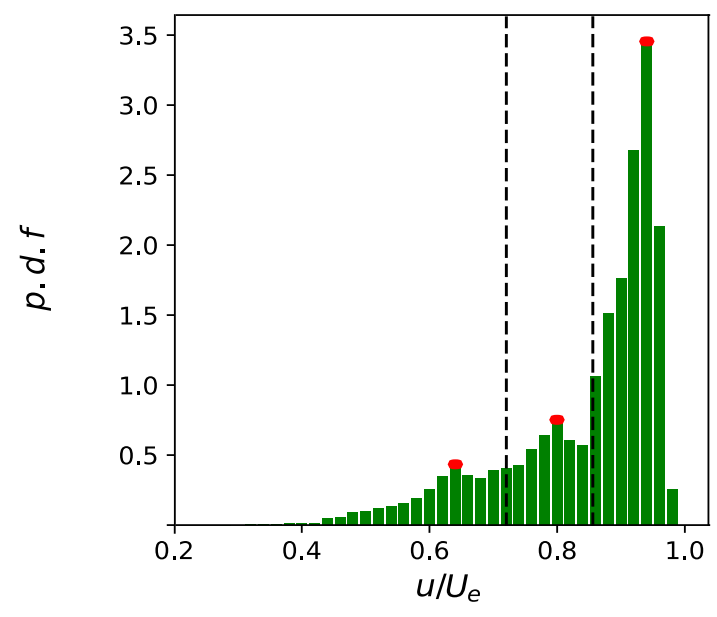

(a)

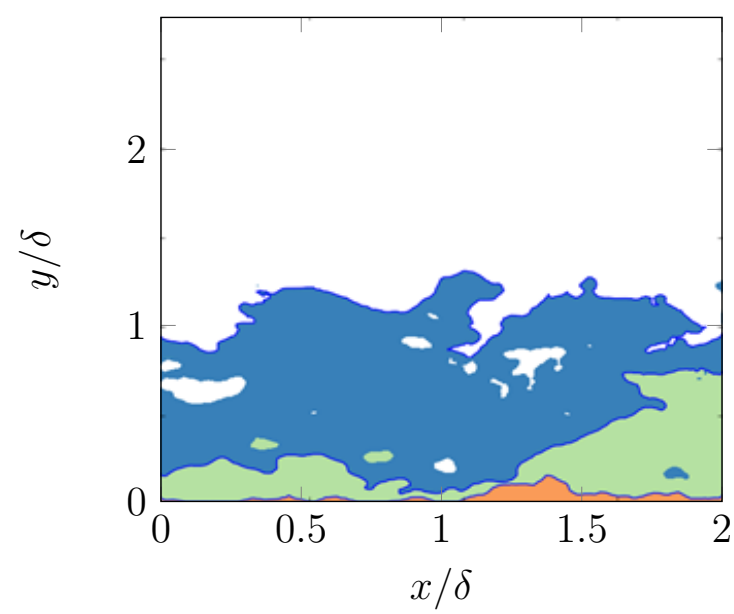

(b)

Figure 2: (a) P.d.f. of normalised instantaneous streamwise velocity field $\left(u / U_{e}\right)$ showing local peaks in the streamwise velocity component (red spots) and the edge velocity of UMZs (dashed line), (b) contours of UMZs detected from the modal velocities, where the solid blue line represents the edges of UMZs

of TNTI, the velocity vectors in the turbulent region in each instantaneous velocity field alone are used to construct the p.d.f. of the streamwise velocity for that instant.

A p.d.f. of the streamwise velocity could also be constructed by ensemble averaging the p.d.f.s across all the instantaneous velocity fields or ensemble averaging the p.d.f. at a single wall-normal position. However, this approach will smooth the pd.f. and remove any remanence of the modal velocities observed in the p.d.f. of the instantaneous streamwise velocity, making it impossible to detect UMZs [Adrian et al., 2000]. Hence, the velocity information from each instantaneous fields is used to generate the p.d.f. of the streamwise velocity and to identify the sample UMZs and compute their sample characteristics.

The process of identifying the UMZs is briefly described based on an example streamwise domain or extent of $2 \delta$. The effect of the size of the streamwsie extent will subsequently be addressed. The p.d.f. of each instantaneous streamwise velocity sample normalised with the local free stream velocity $\left(u / U_{e}\right)$ is computed over a fixed streamwise extent. In the computed p.d.f. of the instantaneous streamwise velocity, the local peaks are located, and their corresponding velocities are identified as the modal velocities as shown in figure $2(a)$, where the peaks are marked with the red spots. The corresponding modal velocities for this example are $0.64 U_{e}, 0.8 U_{e}$ and $0.92 U_{e}$. Thus, the sample number of UMZs identified in this instantaneous velocity field is $N_{u m z}=3$. After the identification of the number of UMZs, the edge velocities are identified as the velocity at the midpoint of the neighbouring modal velocities as shown in figure $2(a)$, where the edge velocities are identified with the vertical black dashed lines. Figure $2(b)$ shows the corresponding contour plot of UMZs in the physical flow domain over the fixed streamwise extent, with the edges of UMZs represented in solid blue lines and the UMZ identified using a uniform colour.

The number of identified UMZs depends on the number of local maxima, i.e. the modal/uniform velocities, detected in the instantaneous p.d.f. of the streamwise velocity. In turn this depends on the streamwise extent of the TBL used to compute the p.d.f. as well as the bin size. Starting with the consideration of the former, the sensitivity of UMZs detection to streamwise extent 
(a)

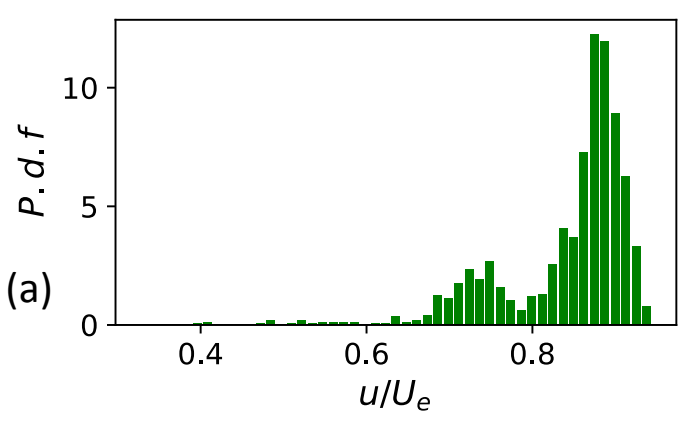

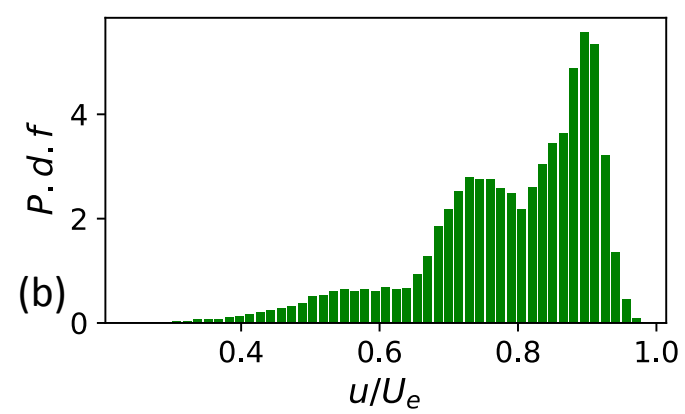

Figure 3: P.d.f. of normalised instantaneous streamwise velocity fields $\left(u / U_{e}\right)$ over a streamwise domain length of $(a) 0.1 \delta,(b) 4 \delta$.
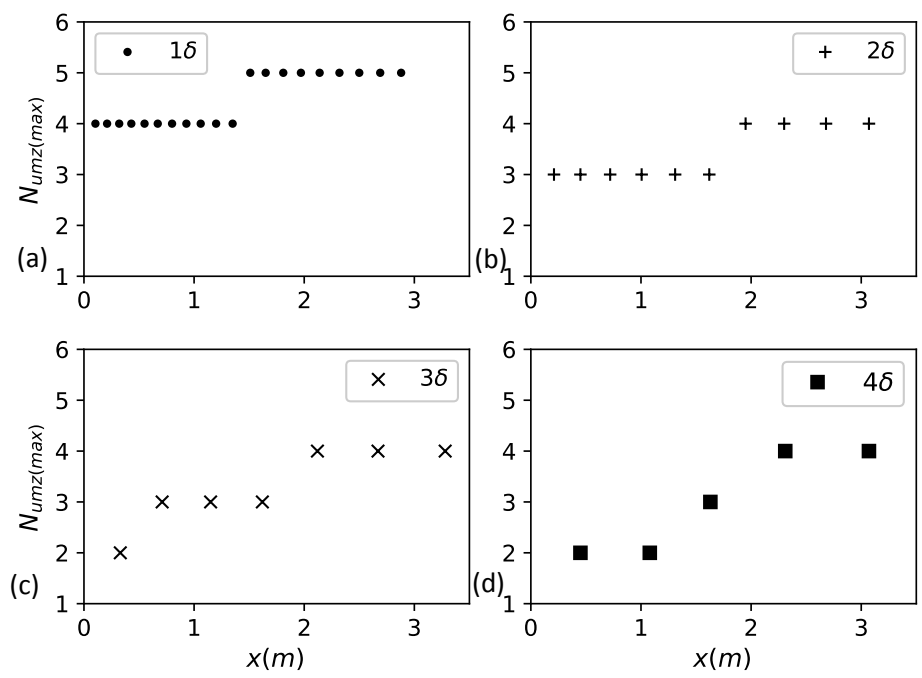

Figure 4: Influence of streamwise domain length, (a) $1 \delta$, (b) $2 \delta$, (c) $3 \delta$ and (d) $4 \delta$ on the number of UMZs detected for 50 bins.

was examined by keeping the number of bins fixed at 50, a number of bins found to be an appropriate compromise in the previous study of UMZs in a ZPG-TBL by de Silva et al. [2016]. Figure $3(a, b)$ shows the variation of modal velocities detected with respect to different streamwise domain extents for a sample instantaneous velocity field. For a small streamwise domain extent of $0.1 \delta$ as shown in figure $3(a)$ a large number of modal velocities are detected, whereas for larger domain extents such as $4 \delta$ shown in figure $3(b)$ the modal velocities are smoothed out and are difficult to identify.

This observation is for a single instantaneous velocity field sample. In order to further examine the sensitivity of UMZs detection to the streamwise domain extent across all available velocity field samples, the maximum number of UMZs for all velocity samples along the APG domain are computed and compared for streamwise domain extents of $1 \delta, 2 \delta, 3 \delta$ and $4 \delta$. Figure 4 shows the influence of streamwise domain extent on the maximum number of UMZs, $N_{u m z(\max )}$, detected across all 30,000 velocity field samples. In the case of a streamwise domain extent of $1 \delta, N_{u m z(\max )}$ ranges between 4 and 5 , whereas for a streamwise domain extent of $4 \delta, N_{u m z(\max )}$ ranges between 2 and 4 . 
(a)

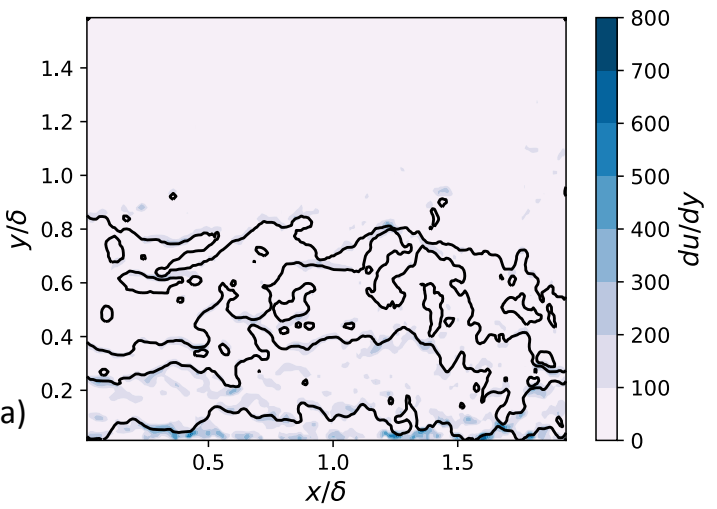

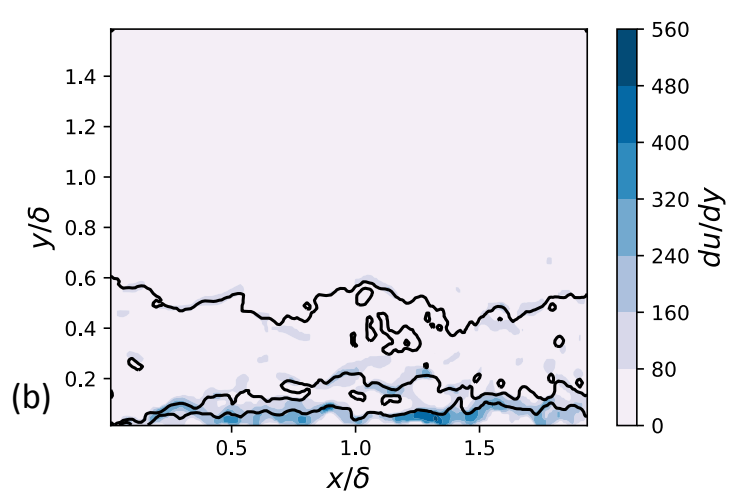

Figure 5: Contour plot showing large $d u / d y$ at the edges of UMZs, (a) 4 UMZs, (b) 3 UMZs.

(a)

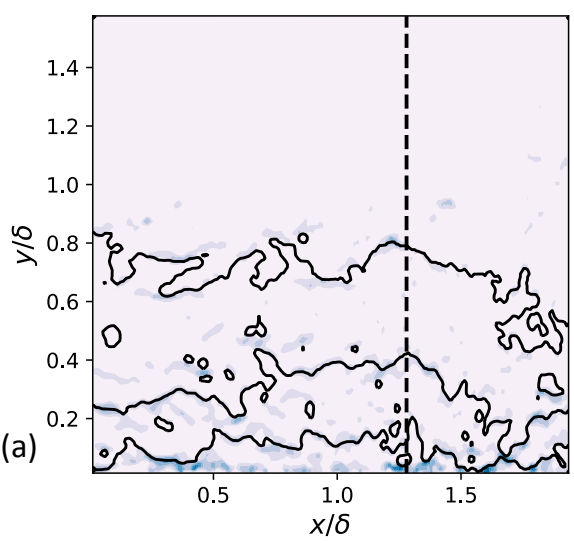

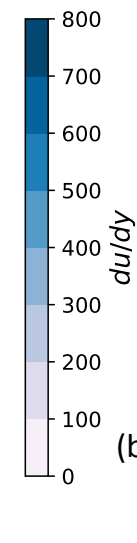

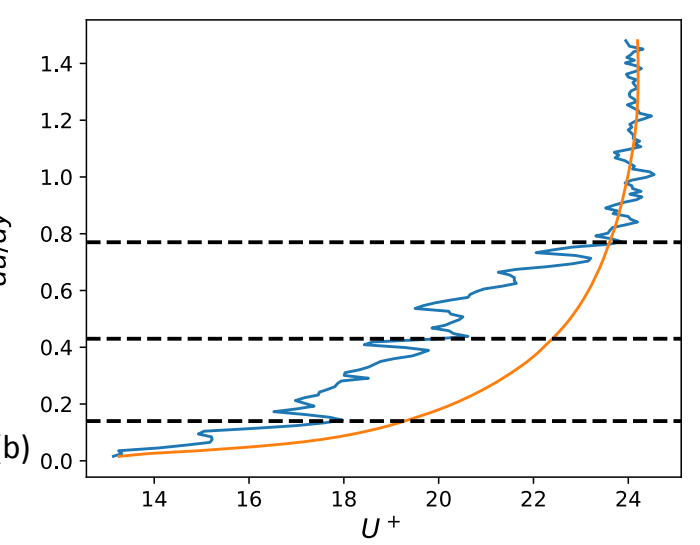

Figure 6: (a) Contour plot showing large $d u / d y$ at the edges of UMZs. (b) Comparison between mean and instantaneous profiles at $x / \delta=1.3$ showing step like jumps from one zone to another.

Figure $5(a, b)$ shows typical contour plot of the wall-normal streamwise velocity gradient, $d u / d y$, for the cases when 3 and 4 UMZs are detected at the same location in the APG domain. The interfaces between UMZs are included in figure $5(a, b)$ and denoted by the solid black lines. A close correspondence is observed between these interfaces and locations of high local values of $d u / d y$. This was also observed for the ZPG-TBL by Adrian et al. [2000]. In order to inspect this behaviour further, consider figure $6(a)$, which shows a typical contour plot of $d u / d y$ in the APG domain for a velocity field which has $3 \mathrm{UMZs}$, with three distinct changes in the streamwise velocity at the interfaces between the UMZs. From this data, the instantaneous streamwise velocity is extracted at the location $x / \delta=1.3$, which is denoted by the vertical dotted line in figure $6(a)$, and graphed in figure $6(b)$. The streamwise velocity is graphed in inner (viscous units), i.e. $U^{+}=U / U_{\tau}$, where $U_{\tau}=\sqrt{\tau_{w} / \rho}$ is the friction velocity, $\tau_{w}$ is mean wall shear stress and $\rho$ is the density. The mean streamwise velocity profile is also graphed in figure $6(b)$ for comparison. Unsurprisingly there are large structural differences between the very smooth mean streamwsie velocity profile and the instantaneous streamwise velocity profile with large wall-normal gradients of the instantaneous streamwise velocity at the UMZ interfaces.

Adrian et al. [2000] used a streamwise domain extent of $1.2 \delta$ to identify UMZs in ZPG- 
TBL, which was the maximum streamwise extent of the their PIV measurements, with no details provided on the number of bins used to compute the histogram of the instantaneous streamwsie velocity used to identify the modal velocities and hence, the UMZs. de Silva et al. [2016] used a streamwise domain extent of $2 \delta$ in their study of UMZs in a ZPG-TBL, which was the maximum streamwise extent of the their PIV measurements, finding no significant variation in the number of UMZs observed for the streamwsie domain extents available to them, i.e. less than $2 \delta$. The sensitivity of determining the modal velocities to the number of bins in the p.d.f. was considered by computing the p.d.f. with 20 bins and 75 bins. The p.d.f. computed with 20 bins was severely smoothed, yet it still indicated the correct number of modal velocities for that sample. This was also the case when 75 bins were used, although in this case significantly more variability in the p.d.f. was found due the limited number of velocity samples that are available for each bin. Therefore, since the detection of the modal velocities was found to be relatively insensitive in the range of 20 to 75 bins and for consistency with the previous work of de Silva et al. [2016] the number of bins was fixed at 50.

To summarise, for our present study given our p.d.f. sensitivity analysis results and for consistency with the more recent study by de Silva et al. [2016], a streamwise domain extent of $2 \delta$ is used with 50 bins in the computation of the p.d.f. of the non-dimensional streamwise velocity.

\section{Characterisation of UMZs in the APG domain}

The characteristics of UMZs based on $R e_{\theta}, \beta$, geometrical properties and flow statistics for both free-stream velocities of $5 \mathrm{~m} / \mathrm{s}$ and $9 \mathrm{~m} / \mathrm{s}$ are presented in this section. The computation of these characteristics is started by dividing the entire APG domain into 10 sub-domains each of $2 \delta$ streamwise extent, where $\delta$ is the local boundary layer thickness for the sub-domain in question.

Using the instantaneous streamwise velocity within the streamwise extent of $2 \delta$ and 50 bins, the instantaneous p.d.f. of the normalised streamwise velocity is computed, the modal velocities in the p.d.f. are identified, which yields the number of UMZs using the methodology presented in section 3 for each of the 30,000 instantaneous velocity fields for each of the 10 sub-domains. The number of UMZs $\left(N_{u m z}\right)$ detected at intervals of $2 \delta$ along the APG domain for $5 \mathrm{~m} / \mathrm{s}$ and $9 \mathrm{~m} / \mathrm{s}$ for all 30,000 velocity field samples are presented in the form of a p.d.f. in figure 7 . The $x$ values in figure 7 represents the midpoint of each $2 \delta$ sub-domain. The probability of an instantaneous velocity field having 3 and 4 UMZs are significantly higher than the velocity fields having either more or less UMZs. For both free stream velocities, up to $\mathrm{x} \approx 1.5 \mathrm{~m}$, the p.d.f. of the number of UMZs peaks at 3 UMZs $\left(N_{u m z(\max )}\right)$ with 4 UMZs becoming more likely further downstream, indicating an increase in the number fo UMZs along the APG domain for both free-stream velocities.

To investigate this observation in more detail, the effect of $\operatorname{Re}_{\theta}$ and $\beta$ on the number of UMZs is examined by representing the $N_{u m z(\max )}$, i.e. the peak in the p.d.f. of the number of UMZs detected as a function of $R e_{\theta}$ and $\beta$. The influence of $R e_{\theta}$ on the UMZs are identified by comparing the $N_{u m z(\max )}$ between the free-stream velocities of $5 \mathrm{~m} / \mathrm{s}$ and $9 \mathrm{~m} / \mathrm{s}$. Figure 8 shows $N_{u m z(\max )}$ represented as a function of $R e_{\theta}$ for both free-stream velocities which shows that the $N_{u m z(\max )}$ increases from 3 UMZs to 4 UMZs. At first, it may appear that this increase depends on Reynolds number, but this is not the case, as the increase in UMZs does not occur at the same $R e_{\theta}$ for both free-stream velocities of $5 \mathrm{~m} / \mathrm{s}$ and $9 \mathrm{~m} / \mathrm{s}$, but rather occurs at a 
Figure 7: P.d.f. of the number of UMZs identified along the APG domain over a streamwise domain length of $2 \delta,(a) 5 \mathrm{~m} / \mathrm{s}$, (b) $9 \mathrm{~m} / \mathrm{s}$. The $x$ value represents the midpoint of each $2 \delta$ sub-domain.

point in space between $x=1.5-1.8 \mathrm{~m}$ for both free-stream velocities.

Table 2 summarizes the comparison of the number of UMZs identified in this study with the previous investigations of ZPG-TBLs. Adrian et al. [2000] reported that for a low to moderate Reynolds number, no significant increase in the number of UMZs is observed. However, for a wide range of Reynolds number, a log-linear increase in the number of UMZs was observed by [de Silva et al., 2016]. For instance, de Silva et al. [2016] reported that at $R e_{\theta}=22,400$, the p.d.f. of the number of UMZs peaks at 4 and it shifts to 5 at $R e_{\theta}=40,800$. In the present study, for the free-stream velocity of $5 \mathrm{~m} / \mathrm{s}$, the peak in the p.d.f. of the number of UMZs shifts from 3 to 4 at $R e_{\theta} \approx 9,000$, whereas for the free-stream velocity of $9 \mathrm{~m} / \mathrm{s}$ the peak in the p.d.f. of the number of UMZs shifts from 3 to 4 at $R e_{\theta} \approx 15,000$. However, the increase in the most likely number of of UMZs cannot be attributed to a $R e_{\theta}$ effect for the Reynolds number range of the present experiments, which is much lower than that in the study by de Silva et al. [2016].

Figure 9 shows the variation of $N_{u m z(\max )}$ as a function of $\beta$ for both free-stream velocities. The increase in $N_{u m z(\max )}$ is observed along the APG domain for both free-stream velocities developing under similar APG at the same spatial location and the same $\beta$ value. The increase in the most likely number of UMZs detected is most likely due to the enhanced growth of an APG-TBL, i.e. larger $\delta$, which is enhanced by increasing $\beta$ Kitsios et al. [2016, 2017]. However, a clear relationship between $\beta$ and $N_{u m z(\max )}$ cannot be established since $\beta$ varies as the flow develops with varying $R e_{\theta}$ in the streamwise direction and their interaction cannot be completely decoupled. 


\subsection{Influence of $N_{u m z}$ on modal velocity}

The number of UMZs identified equates to the number of modal velocities detected, and as the number of UMZs increases, the magnitude of the modal velocities varies. To investigate how the modal velocities detected vary with the number of UMZs, the modal velocity associated with each UMZ in the velocity fields having 2,3,4,5 or 6 UMZs are compared within the same $2 \delta$ domain. It is important to note that the velocity fields having a single UMZ are not included as their contribution to the total velocity fields is significantly less compared to other velocity fields as shown in figure 7 . The modal velocities detected in each instantaneous velocity field are grouped based on the number of UMZs detected, i.e. 2, 3, 4, 5 or 6 UMZs, and averaged to obtain mean modal velocities, which are represented as $U_{m-i}$, where $i$ represents the number of modal velocities or the number of UMZs present in the instantaneous velocity field. For example, modal velocities associated with each UMZ in the velocity field having three

Figure 8: Peak in the p.d.f. of the number of UMZs $\left(N_{u m z(\max )}\right)$ detected represented as a function of Reynolds number $R_{\theta},(a) 5 \mathrm{~m} / \mathrm{s},(b) 9 \mathrm{~m} / \mathrm{s}$. The $x$ value represents the midpoint Table 2: A review of previous studies on the number of UMZs

\begin{tabular}{lllllll}
\hline Particulars & $U_{\infty}$ & $U_{\tau}$ & $R e_{\theta}$ & $R e_{\tau}$ & $\begin{array}{l}\text { No. of } \\
\text { realization }\end{array}$ & $\begin{array}{l}\text { Peak in the p.d.f. of number of UMZs } \\
\left(N_{u m z(m a x)}\right)\end{array}$ \\
\hline $\begin{array}{l}\text { Adrian et al. [2000] } \\
\text { (ZPG) }\end{array}$ & 10.8 & 0.4 & 6845 & 2000 & 65 & 3 (no significant increase) \\
$\begin{array}{l}\text { de Silva et al. [2016] } \\
\text { (ZPG) }\end{array}$ & 10 & 0.33 & 22400 & 8000 & 1440 & 4 \\
$\begin{array}{l}\text { de Silva et al. [2016] } \\
\text { (ZPG) }\end{array}$ & 20 & 0.63 & 40800 & 14500 & 1440 & 5 \\
$\begin{array}{l}\text { Present Study } \\
(5 m / s)(A P G)\end{array}$ & 5 & 0.32 & $4000-12000$ & 2400 & 30000 & Increased from 3 to 4 \\
$\begin{array}{l}\text { Present Study } \\
(\text { APG })(9 m / s)\end{array}$ & 9 & 0.6 & $6000-22000$ & 4200 & 30000 & Increased from 3 to 4 \\
\hline
\end{tabular}




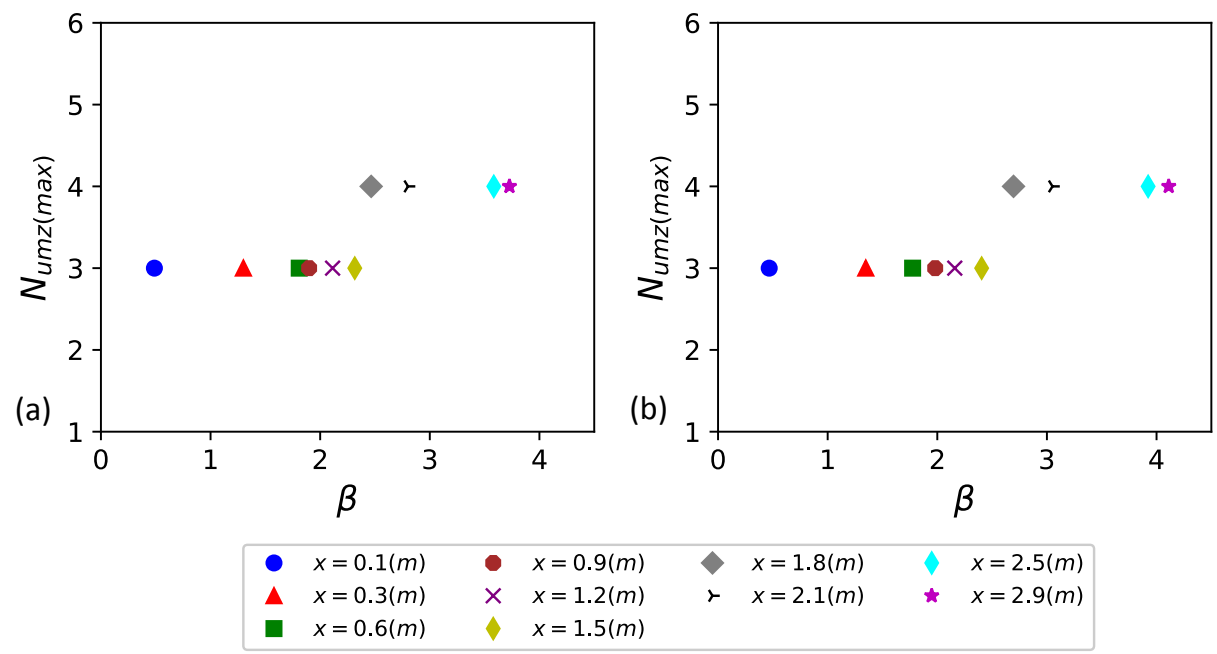

Figure 9: Peak in the p.d.f. of the number of UMZs $\left(N_{u m z(\max )}\right)$ detected represented as a function of non-dimensional pressure gradient parameter $\beta,(a) 5 \mathrm{~m} / \mathrm{s},(b) 9 \mathrm{~m} / \mathrm{s}$. The $x$ value represents the midpoint of each $2 \delta$ domain.

UMZs are represented as $U_{m 1}, U_{m 2}$ and $U_{m 3}$, where $U_{m 1}$ represents the highest modal velocity, or the modal velocity detected closest to the free-stream, while the others are represented in descending order respectively as shown in figure $10(a)$.

Figure $10(b)$ shows the distribution of mean modal velocities as a function of the $N_{u m z}$ at $x=1.2 \mathrm{~m}$ for $5 \mathrm{~m} / \mathrm{s}$. With increasing number of UMZs, the mean modal velocities of existing UMZs move to higher values to accommodate new modal velocities, i.e. new UMZs that develop near the wall. The modal velocities closer to the wall show a relatively larger increase in velocity as new UMZ develop while the modal velocities of the UMZs closest to the free-stream, i.e. $U_{m 1}$ show a relatively smaller increase. This suggests that as the $N_{u m z}$ increases, more UMZs appear without significantly altering the largest modal velocity, i.e. modal velocity of the UMZ closest to the free-stream. This observation is consistent across all other $2 \delta$ sub-domains of the APG region for the free-stream velocity of $5 \mathrm{~m} / \mathrm{s}$ as well as for the free-stream velocity of 9 $\mathrm{m} / \mathrm{s}$ as shown in figure $10(\mathrm{c})$. The development of new UMZ closer to the wall is in agreement with the attached eddy model of Townsend [1976], Head and Bandyopadhyay [1981], Perry and Chong [1982], which indicates that the vortical structures scale with wall-normal distance and hence, have their origin at the wall, as well as the vortical model and its relationship to UMZ proposed by Adrian et al. [2000].

\subsection{Influence of $N_{u m z}$ on thickness of UMZs}

In addition to the characterisation of UMZs by their modal velocities, the UMZs are also characterised by their geometrical property such as UMZ thickness $\left(t_{u m z}\right)$. The thickness of a UMZ is defined as the wall-normal distance between the upper and lower edge of the UMZ. Initially, the wall-normal distance from the wall to the upper edges of each UMZ is determined, which will be referred to as the wall-normal thickness. Figure 11 shows an instantaneous velocity field having 3 UMZs, where the wall-normal thickness of the UMZ closest to the free-stream 

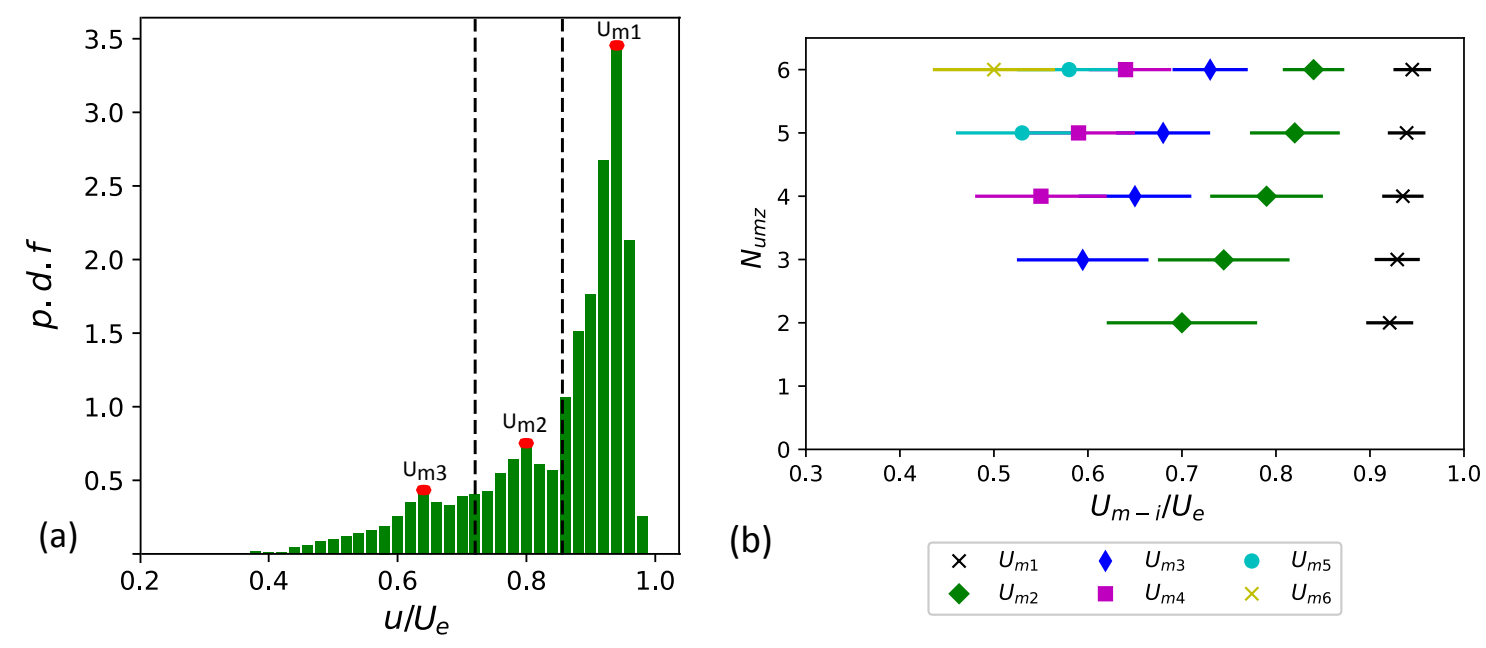

(b)
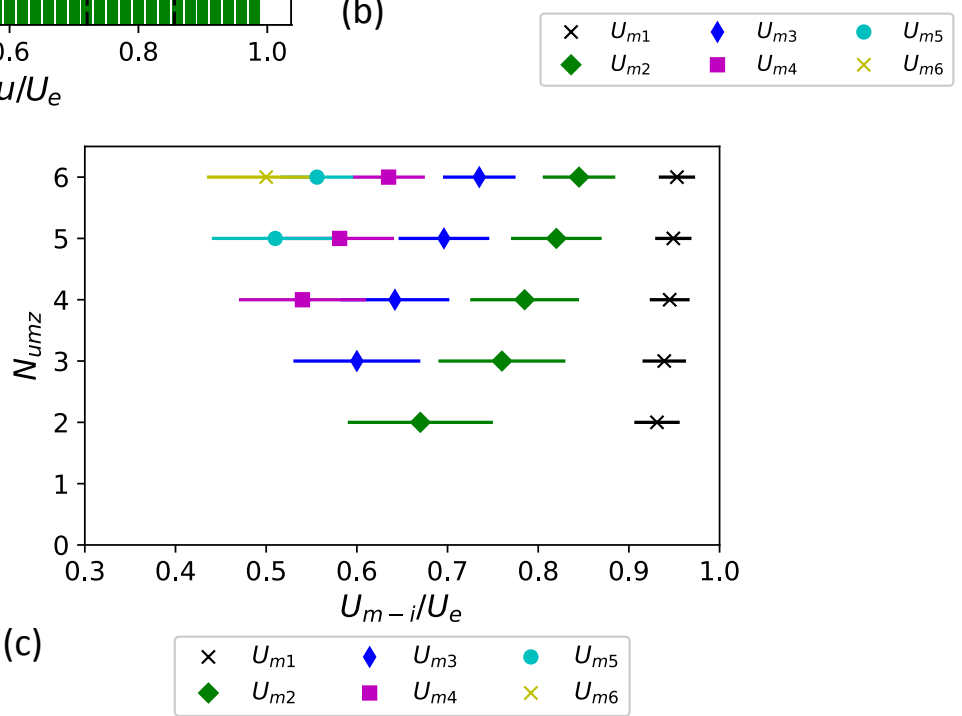

Figure 10: (a) Representation of modal velocities, $(b, c)$ Variation of mean modal velocities as a function of $N_{u m z}$ for a particular $2 \delta$ domain at $x=1.2 \mathrm{~m}$ for $5 \mathrm{~m} / \mathrm{s}$ and $9 \mathrm{~m} / \mathrm{s}$ respectively. Error bar represents the standard deviation of the mean modal velocities.

is represented as $W t_{u m z 1}$ and others are represented in ascending order, i.e. the UMZ below the UMZ closest to the free-stream is represented as $W t_{u m z 2}$ with the UMZ closest to the wall is represented as $W t_{u m z 3}$. The thickness of each UMZ is then determined by subtracting the wall-normal thickness of neighbouring UMZs, i.e. $t_{u m z 1}=W t_{u m z 1}-W t_{u m z 2}$ and $t_{u m z 2}=W t_{u m z 2}$ - $W t_{u m z 3}$ and $t_{u m z 3}=W t_{u m z 3}$.

Once the thicknesses of all UMZ in the 30,000 velocity samples have been identified, subsets of velocity fields conditioned on the number of UMZs, $N_{u m z}$, are extracted and used to compute the p.d.f. of the thicknesses of each UMZ for that subset. Within each subset, the mean thickness of each UMZ is obtained from the p.d.f. of the thickness of each UMZ. Figure 12 shows the p.d.f. of the thicknesses of UMZs for the subset of velocity fields with $N_{u m z}=3$ and 4 at $x=1.2 \mathrm{~m}$ for $5 \mathrm{~m} / \mathrm{s}$, where each colour represents the thickness of each UMZ. The thickness of UMZs increases with increasing wall-normal distance, which can be seen by the shift in the p.d.f. from left to right. From the distribution of p.d.f., it is also evident that the standard deviation of the thickness of UMZs increases with wall-normal position, i.e. the thickness of the UMZ closest to the free-stream has the highest standard deviation compared to the UMZs closer to the wall. 
(a)

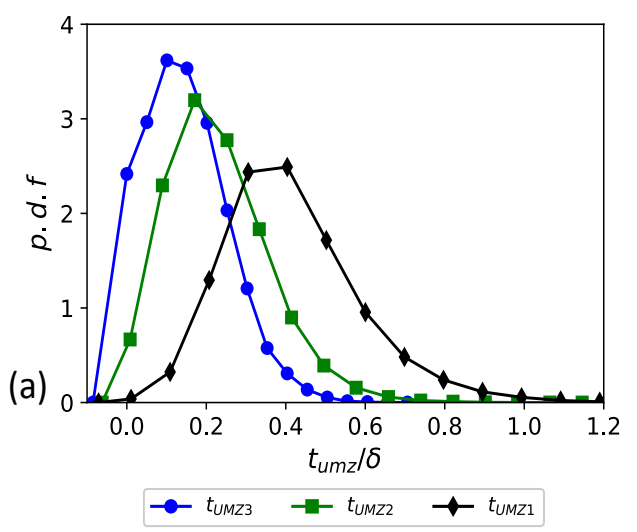

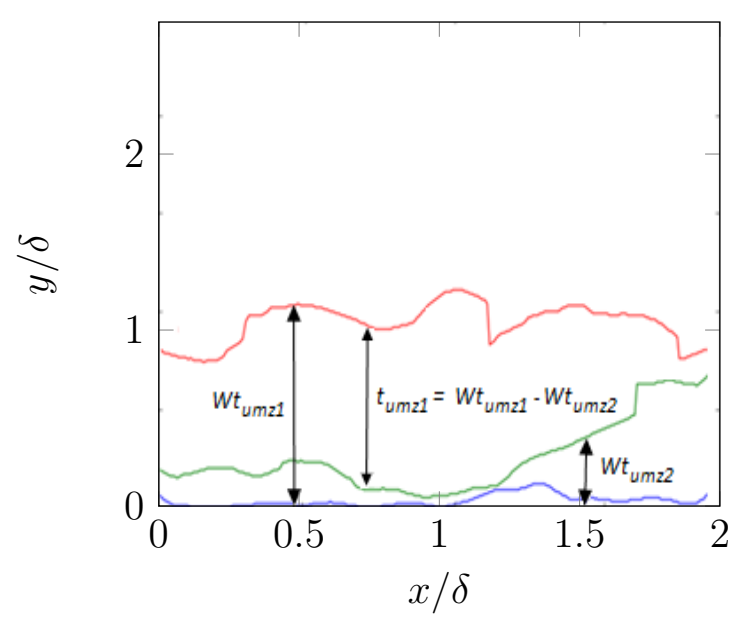

Figure 11: Representation of thicknesses of UMZs.

Figure 12: P.d.f. of UMZ thicknesses at $x=1.2 \mathrm{~m}$ for free-stream velocity of $5 \mathrm{~m} / \mathrm{s},(a) N_{u m z}$ $=3$, blue line - UMZ closest to the wall $\left(t_{u m z 3}\right)$, green line - UMZ above $t_{u m z 3}\left(t_{u m z 2}\right)$, black line - UMZ closest to the free-stream $\left(t_{u m z 1}\right),(b) N_{u m z}=4$, pink line - UMZ closest to the wall $\left(t_{u m z 4}\right)$, blue line - UMZ above $t_{u m z 4}\left(t_{u m z 3}\right)$, green line - UMZ below the UMZ closest to the free-stream $\left(t_{u m z 2}\right)$, black line - topmost UMZ $\left(t_{u m z 1}\right)$.

In order to investigate how the thickness of UMZs varies with $N_{u m z}$, the wall-normal thickness and the thickness of UMZs in velocity fields having $N_{u m z}=2,3,4,5$ and 6 are compared at the same $2 \delta$ sub-domain. Figure $13(a)$ shows the variation of mean wall-normal thickness of each UMZ, i.e. the mean wall-normal location of each UMZ interface, as a function of $N_{u m z}$ at $x=1.2 \mathrm{~m}$ for the free-stream velocity of $5 \mathrm{~m} / \mathrm{s}$. It is evident that there is an increase in the wall-normal location of UMZs with increasing $N_{u m z}$. This supports the observation from the modal velocity characterisation in section 4.1 that as the $N_{u m z}$ increases, new UMZs develop close to the wall, causing the wall-normal location of existing UMZs to move further away from the wall. However, no significant difference in the wall-normal location of UMZ closest to the free-stream, $W t_{u m z 1}$, is observed between velocity fields having a low or high number of UMZs. This suggests that when the $N_{u m z}$ increase, UMZs are more closely packed which in turn reduces (or compresses) the thickness of each UMZ. Figure 13 (b) shows the comparison of mean thickness of each UMZ as a function of $N_{u m z}$ at $x=1.2 \mathrm{~m}$, with the mean thickness of each 
(a)

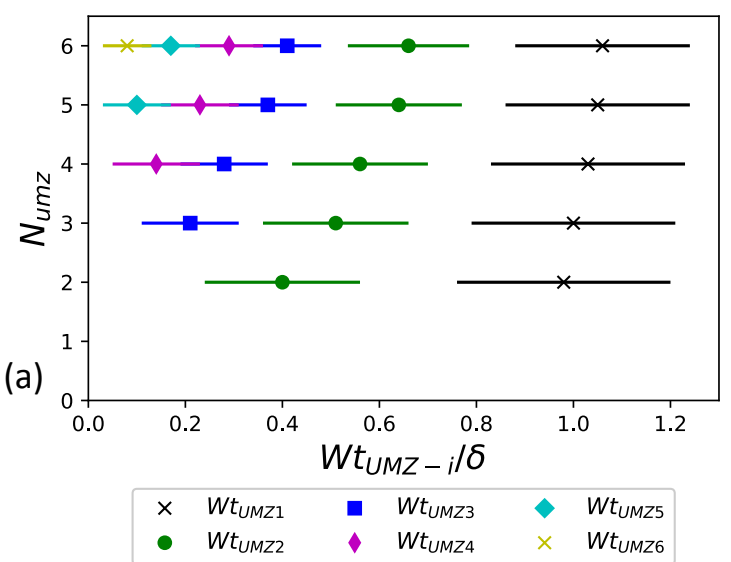

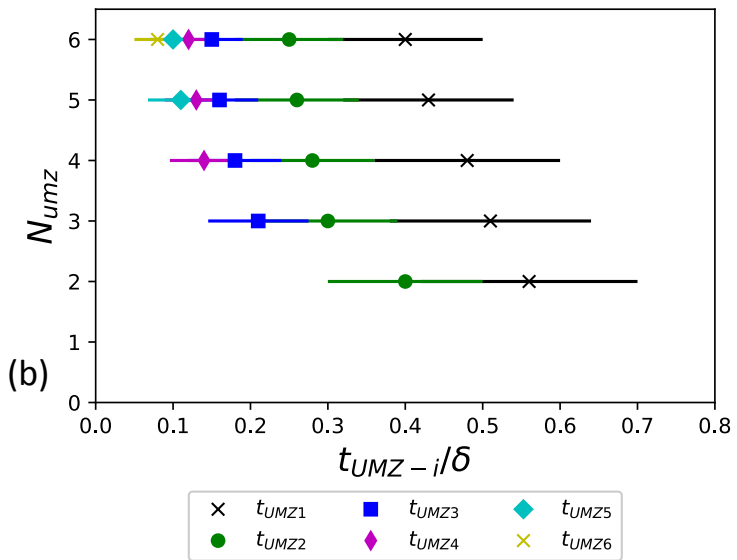

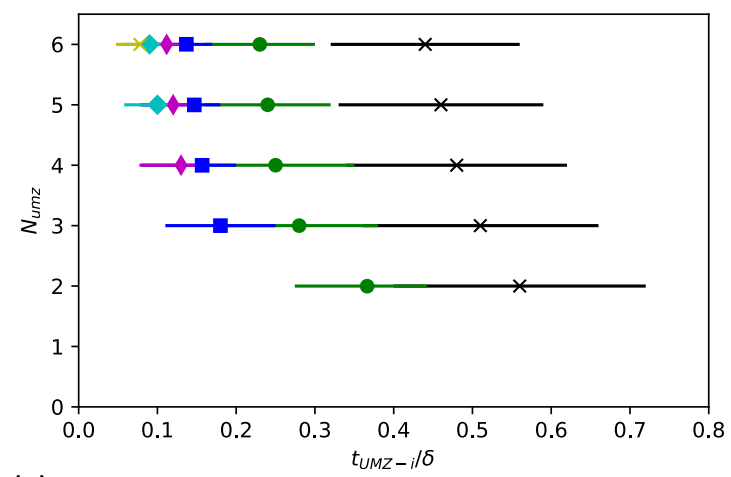

(c)

$\times \begin{array}{llllll}\times & t_{U M Z 1} & t_{U M Z 3} & & \\ \bullet & t_{U M Z 2} & t_{U M Z 4} & \times & t_{U M Z 6} \\ & & & & \end{array}$

Figure 13: Comparison of thickness of each UMZ at $x=1.2 \mathrm{~m}$ as a function of $N_{u m z}(a)$ mean wall-normal thickness for free-stream velocity of $5 \mathrm{~m} / \mathrm{s}),(b, c)$ mean thickness of each UMZ for free-stream velocity of $5 \mathrm{~m} / \mathrm{s}$ and $9 \mathrm{~m} / \mathrm{s}$ respectively. Error bars represent the standard deviation.

UMZ decreasing with increasing $N_{u m z}$ from 2 to 6 . These characterisations are consistent in all other $2 \delta$ APG sub-domains for the free-stream velocity of $5 \mathrm{~m} / \mathrm{s}$, as well as for the free-stream velocity of $9 \mathrm{~m} / \mathrm{s}$ as shown in figure $13(\mathrm{c})$.

\subsection{Influence of $N_{u m z}$ on UMZ edge characteristics}

From figure 5 , it is evident that large $d u / d y$ tends to occur at the edges of interfaces between UMZs. In order to investigate this characteristic in more detail, additional characterisations of the interfaces between UMZ based on $d u / d y$ has been conducted. For this task, the interface of each UMZ detected is taken as a reference interface, and its corresponding streamwise velocity, i.e. the edge velocity of the interface and its wall-normal location will be represented as $u_{j}$ and $y_{j}$, respectively. Then conditional statistics below and above the reference interface are determined by identifying the wall-normal position $\left(y_{i}\right)$ and its corresponding streamwise velocity $\left(u_{i}\right)$. This is illustrated in figure 14, showing a single instantaneous velocity field having 3 UMZs, where the red diamond shaped symbol represents the reference interface of UMZ closest to the free-stream, and the yellow diamond-shaped symbol representing the locations 


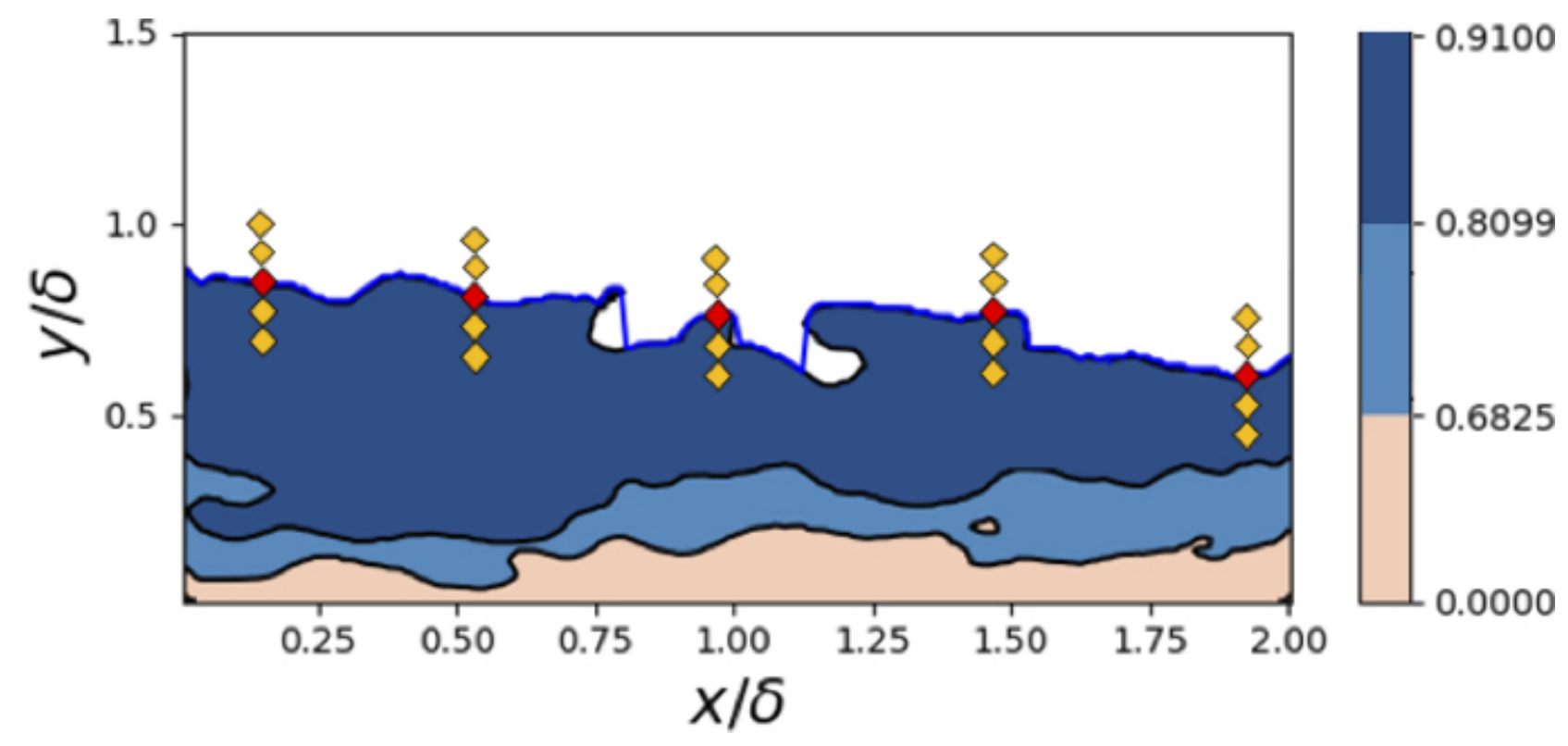

Figure 14: Contour plot showing an instantaneous velocity field having $N_{u m z}=3$, solid blue line - represents the outer edges of the topmost UMZ interface having a single wall-normal location at each streamwise grid, red diamond-shaped symbol - represents the topmost UMZ interface detected (reference interface), yellow diamond-shaped symbol - represents the interfaces above and below the detected/reference interface.

above and below the reference interface between two UMZs. Here the interface of UMZs are taken as the outermost boundary of each UMZ having a single wall-normal position at each streamwise position. This is done to avoid the presence of multiple edges of UMZ at the same location since the interfaces can fold back on themselves as illustrated in figure 14, where the solid black line represents the interface, and the solid blue line represents the interface having a single wall-normal location at each streamwise location.

The wall-normal positions $\left(y_{i}\right)$ and streamwise velocities $\left(u_{i}\right)$ at seven points above and seven points below the reference interface are determined at each streamwise position of the interface between UMZs. The conditional profile of $u$ is computed at each streamwise position along the interface as a function of the coordinate $y_{j}-y_{i}$. Then, the conditional profiles obtained at each streamwise location along the interface between the UMZs are averaged along the interface in the streamwise extent of $2 \delta$, which yields the conditionally averaged streamwise velocity profile across the interface between the UMZs. In order to compare the conditional statistics at between the different UMZ interfaces, the same procedure is repeated for the different UMZ interface.

Figure $15(a, c)$ shows the variation in the conditionally averaged profile of streamwise velocity across the interface of each UMZ in two single instantaneous velocity field having $N_{u m z}=3$ and 4 . The interface between two UMZs is denoted as $I_{i}$, where $i$ represents the number of the UMZs interface present in the instantaneous velocity field starting with the one closest to the free-stream. For example, the interface associated with each UMZ in the velocity field having $N_{u m z}=4$ is represented as $I_{1}, I_{2}, I_{3}$ and $I_{4}$, where $I_{1}$ represents the interface of the UMZ closest to the free-stream, i.e. TNTI, and others are represented in ascending order respectively such that $I_{4}$ represents the UMZ interface closest to the wall. The conditionally averaged profile of the streamwise velocity obtained across the UMZ interface enables us to 

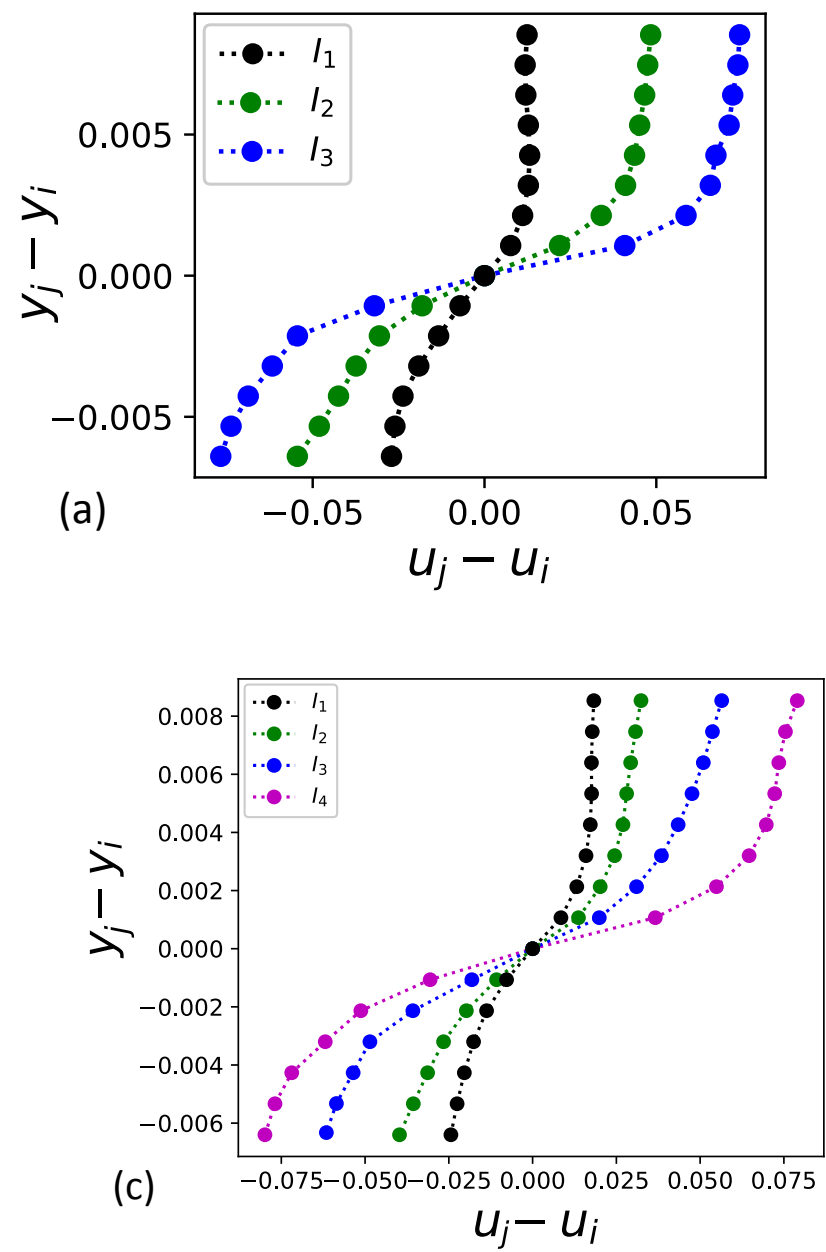

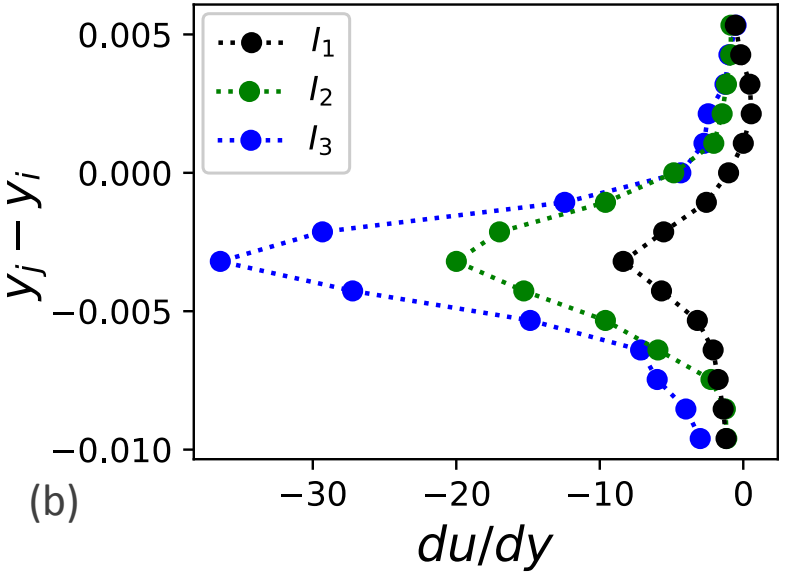

(d)

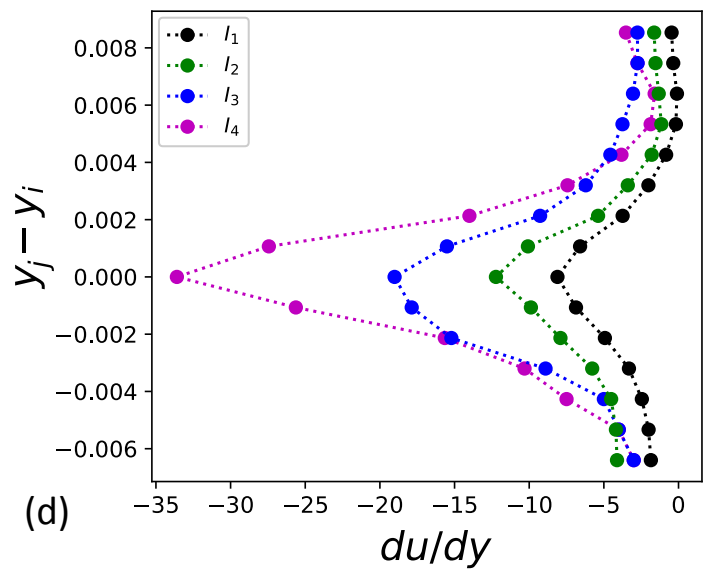

Figure 15: Profile of the streamwise velocity, $u$, across the interface between UMZs in a single instantaneous velocity field having $(a) N_{u m z}=3,(c) N_{u m z}=4 . d u / d y$ across the interfaces between UMZs in a single instantaneous velocity field having $(b) N_{u m z}=3,(d) N_{u m z}=4 . u_{j}$ and $y_{j}$ denote the velocity and wall-normal position of the detected interface/reference interface, whereas $u_{i}$ and $y_{i}$ denote the velocities and wall-normal positions above and below the detected interface.

characterise the interface based on the jump in streamwise velocity across the UMZ interface $\left(\Delta_{u}\right)$ and the width of the UMZ interface $\left(\delta_{w}\right)$ as shown in figure 16 .

Comparing the conditionally averaged velocity profiles among the UMZ interface shows that $\Delta_{u}$ decreases with increasing wall-normal position as shown in figure $15(a, c)$, where the UMZ interface closest to the free-stream, $I_{1}$, has the smallest jump in streamwise velocity compared to the UMZ interface closest to the wall, i.e. $I_{3}$, for $N_{u m z}=3$ or $I_{4} N_{u m z}=4$. Figure 15 $(b, d)$ shows the variation in $d u / d y$ across the UMZ interface with the UMZ interface closest to the wall having the largest velocity gradient, $d u / d y$. This larger streamwise velocity gradient noticed at the UMZ interface closest to the wall is in agreement with the results shown in the figure 5 and is consistent with the fact that the highest gradient in velocity are observed closer to the wall in a TBL. However, these observations are only for single instantaneous velocity field samples having 3 and $4 \mathrm{UMZs}$, in order to provide statistical significance to these observations this analysis is applied to all corresponding velocity subsets with either $N_{u m z}=3$ or 4 and the 


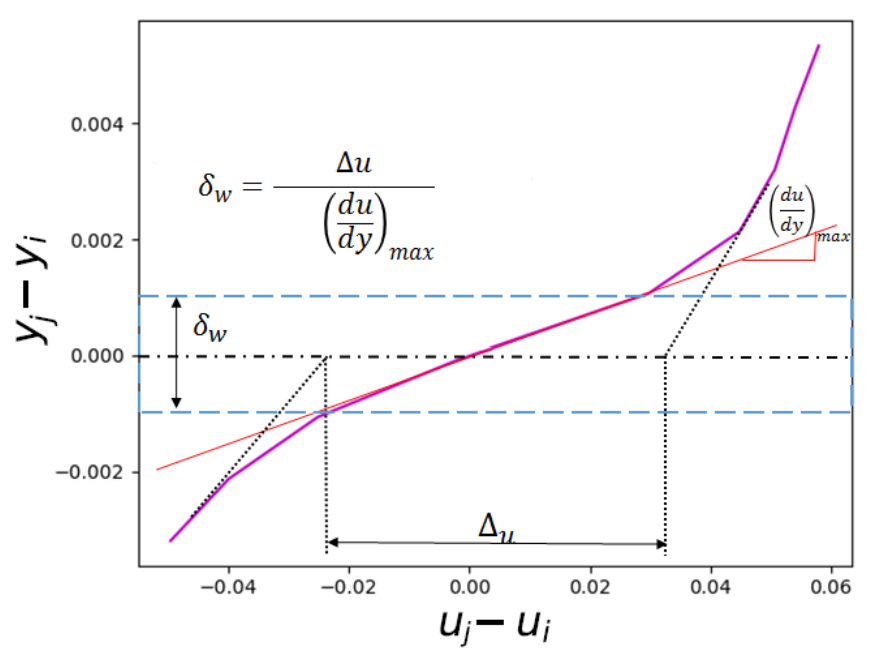

Figure 16: Schematic representation of the jump in streamwise velocity $\left(\Delta_{u}\right)$ across the UMZ interface and the width of the UMZ interface $\left(\delta_{w}\right)$.
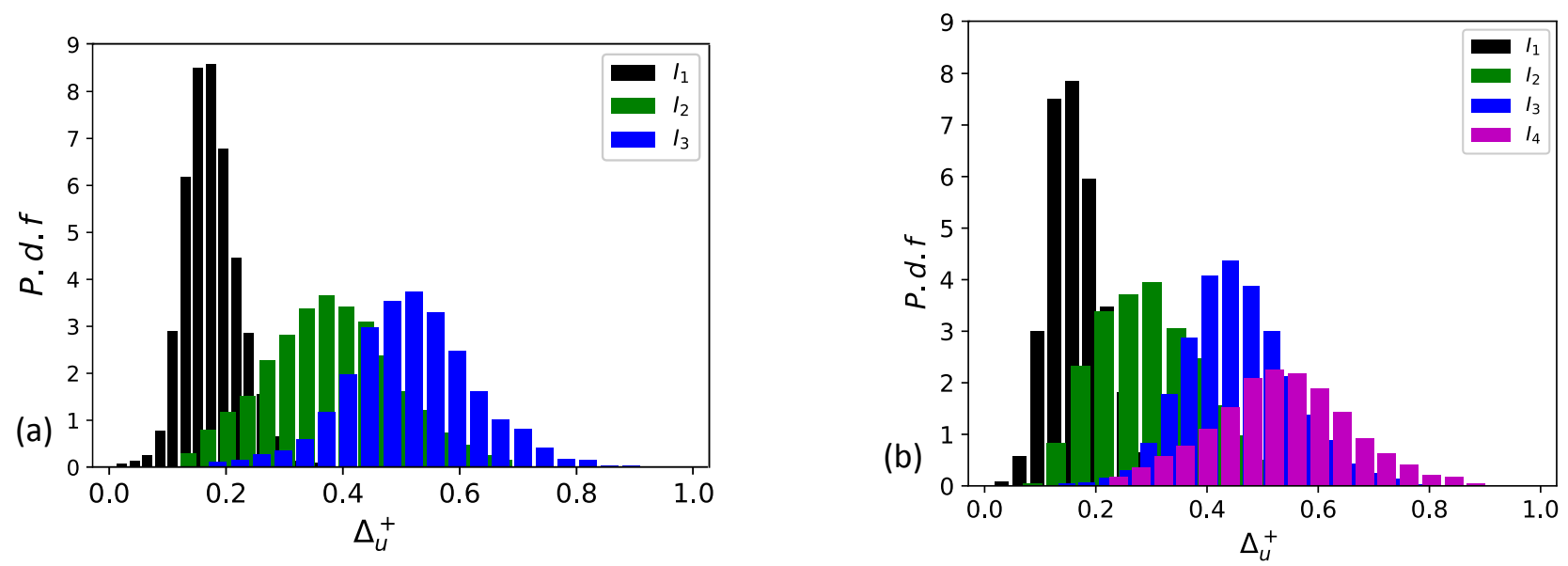

Figure 17: P.d.f. of $\Delta_{u}$ across each UMZ interface in the velocity fields with $(a) N_{u m z}=3$, (b) $N_{u m z}=4$, at $x=0.6 \mathrm{~m}$ for a free-stream velocity of $5 \mathrm{~m} / \mathrm{s}$.

conditional averaged statistics are computed for all other velocity fields belonging to these to subsets.

Figure $17(a, b)$ shows the p.d.f. of $\Delta_{u}$ across each UMZ interface for velocity fields having 3 and $4 \mathrm{UMZs}$ at $x=0.6 \mathrm{~m}$. Consistent with the observation from single sample, a larger $\Delta_{u}$ is observed at the UMZ interface that is closer to the wall compared to the UMZ interfaces that are further away from the wall. To investigate how $\Delta_{u}$ varies with $N_{u m z}, \Delta_{u}$ is determined across each UMZ interface in the subsets of the instantaneous velocity fields with $N_{u m z}=2,3$, 4, 5 and 6 , and the mean values of $\Delta_{u}$ are compared at the same $2 \delta$ domain. Figure 18 shows the distribution of mean $\Delta_{u}$ as a function of $N_{u m z}$ at $x=1.2 \mathrm{~m}$ for a free-stream velocity of $5 \mathrm{~m} / \mathrm{s}$. As $N_{u m z}$ increases, $\Delta_{u}$ across each UMZ interface decreases. This decrease in $\Delta_{u}$ with increasing $N_{u m z}$ is most likely due to the development of new UMZs near the wall causing the interface of the existing UMZs to move away from the wall resulting in a decreased $\Delta_{u}$ across the UMZ interface. Particularly, the UMZ interface closest to the free-stream, i.e. $I_{1}$, shows 

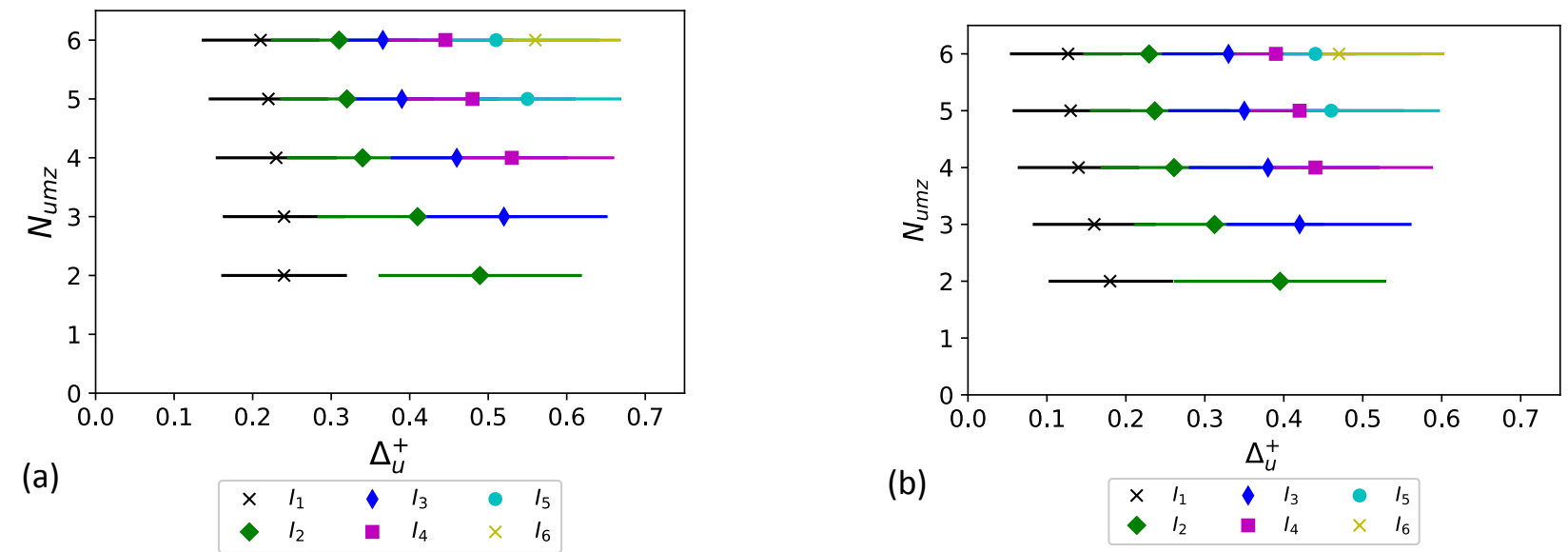

Figure 18: Variation of mean jump in streamwise velocity across the UMZ interface as a function of $N_{u m z}$ at x=1.2 $\mathrm{m}$ for $(a)$ free-stream velocity of $5 \mathrm{~m} / \mathrm{s}$ and $(b)$ free-stream velocity of $9 \mathrm{~m} / \mathrm{s}$. Error bars represent the standard deviation.
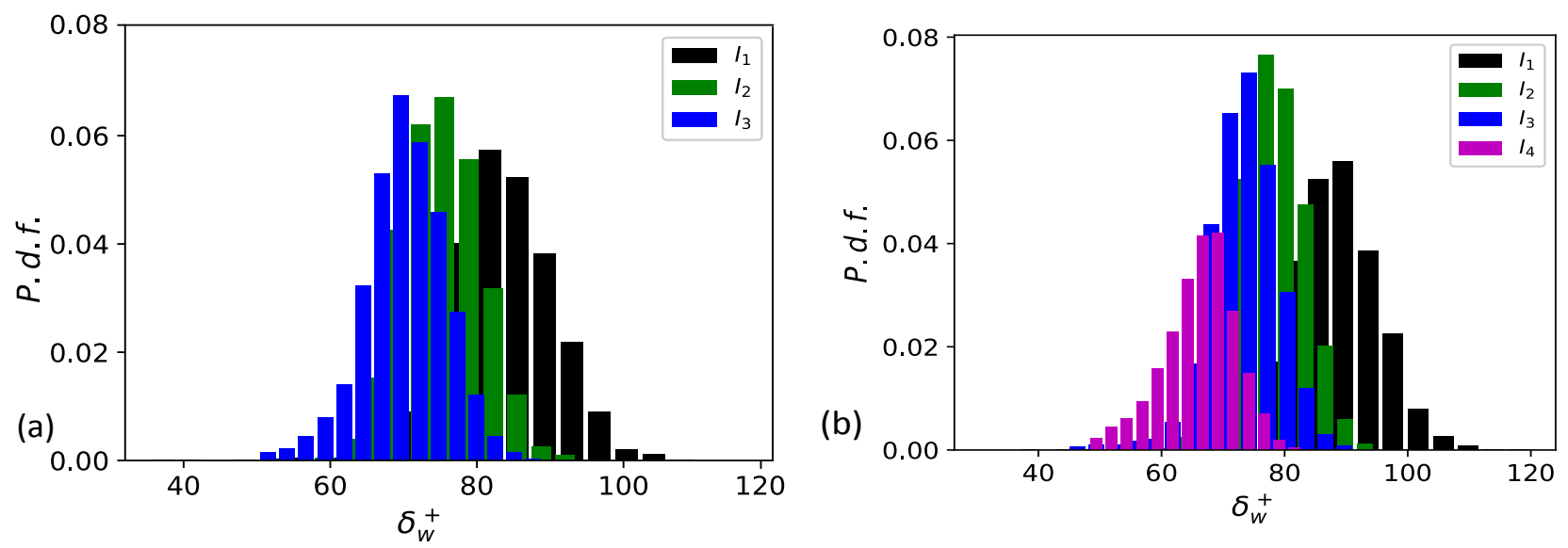

Figure 19: P.d.f. of the width of the UMZ interface $\left(\delta_{w}\right)$ across each UMZ interface in the velocity fields having (a) $3 \mathrm{UMZs,}(b) 4 \mathrm{UMZs}$, at $x=0.6 \mathrm{~m}$ for a free-steam velocity of $5 \mathrm{~m} / \mathrm{s}$.

only a marginal decrease in $\Delta_{u}$ since the wall-normal location of this UMZ interface is not significantly changed with increasing $N_{u m z}$ as shown in figure $13(a)$. These observations are found to be consistent across all other $2 \delta$ APG streamwise domains for the free-stream velocity of $5 \mathrm{~m} / \mathrm{s}$, as well as for the free-stream velocity of $9 \mathrm{~m} / \mathrm{s}$ as shown figure $18(b)$.

From the conditionally averaged velocity profiles, it is also evident that the jump in streamwise velocity occurs across a small wall-normal distance between UMZs. This wall-normal distance is referred as the width of the UMZ interface $\left(\delta_{w}\right)$ [de Silva et al., 2016] and is computed following Chauhan et al. [2014] as,

$$
\delta_{w}=\frac{\Delta_{u}}{(d u / d y)_{\max }}
$$

Figure $19(a, b)$ shows the p.d.f. of $\delta_{w}$ for instantaneous velocity field samples having 3 or 4 UMZs at a $2 \delta$ APG streamwise domain located at $x=0.6 \mathrm{~m}$. These results show that $\delta_{w}$ increases with increasing wall-normal distance, i.e. the outermost UMZ has the largest interface 


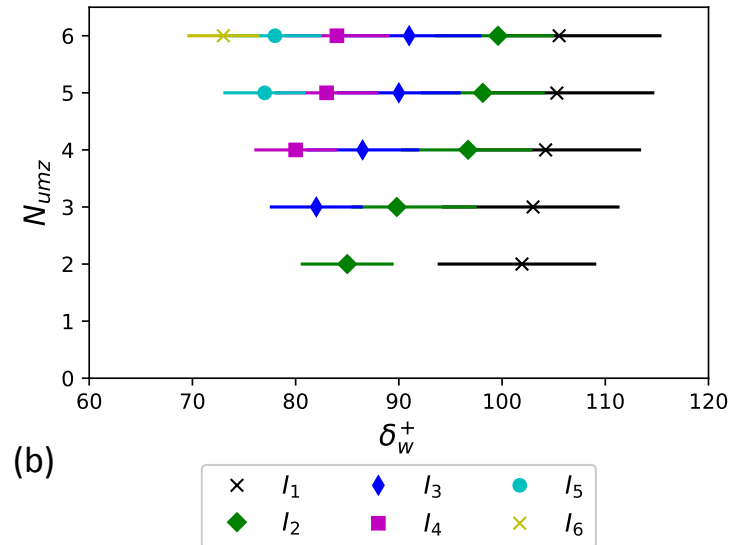

Figure 20: Variation of mean width of UMZ interface as a function of $N_{u m z}$ at $x=1.2 \mathrm{~m}$ for (a) free-stream velocity of $5 \mathrm{~m} / \mathrm{s}$ and $(b)$ free-stream velocity of $9 \mathrm{~m} / \mathrm{s}$. Error bars represent the standard deviation.

width compared to the UMZ interface closest to the wall.

In order to identify the influence of $N_{u m z}$ on the width of UMZ interface, the mean values of $\delta_{w}$ are compared between the velocity fields having $N_{u m z}=2,3,4,5$ and 6 at the same $2 \delta$ APG streamwise domain. Figure 20 shows the distribution of mean $\delta_{w}$ as a function of $N_{u m z}$ at $x=1.2 \mathrm{~m} . \delta_{w}$ across each UMZ interface increases with increasing $N_{u m z}$, which is suggested to be due to the movement of UMZ interface away from the wall with increasing $N_{u m z}$ where the magnitude of $(d u / d y)_{\max }$ is reduces faster compared to $\Delta_{u}$ resulting in increased $\delta_{w}$ (since $\left.\delta_{w}=\Delta_{u} /\left((d u / d y)_{\max }\right)\right)$. This observed variation of $\delta_{w}$ is consistent across all other $2 \delta$ APG domains for the free-stream velocity of $5 \mathrm{~m} / \mathrm{s}$, as well as for the free-stream velocity of $9 \mathrm{~m} / \mathrm{s}$ as illustrated in figure $20(b)$.

\subsection{Variation of geometrical properties of UMZs along the APG domain}

In order to investigate the development of the thicknesses of UMZs, the jump in the streamwise velocity across the UMZ interfaces and width of the UMZ interfaces in the streamwise direction along the APG domain, the p.d.f.s of the thicknesses of UMZs, $\Delta_{u}$ and $\delta_{w}$ corresponding to the subsets with $N_{u m z}=3$ and 4 are computed at five locations corresponding to $x=0.1 \mathrm{~m}, 0.6 \mathrm{~m}$, $1.2 \mathrm{~m}, 1.8 \mathrm{~m}$ and $2.5 \mathrm{~m}$, where $x$ represents the midpoint of each $2 \delta$ streamwise extent along the APG domain. Note that $N_{u m z}=3$ and 4 are selected for this analysis since the occurrence of 3 and 4 UMZs is significantly higher than any others numbers of UMZs.

Figure 21 shows the variation of the mean thicknesses of the UMZs along the APG domain for a free-stream velocity of $5 \mathrm{~m} / \mathrm{s}$. Since the mean wall-normal location of TNTI is approximately equal to $\delta$ as discussed in Appendix A.2 and shown in figure 28, the thickness of UMZ is normalised by the local $\delta$. The increase in the UMZ thickness with wall-normal distance is consistent throughout the APG domain for velocity fields having 3 and 4 UMZs. This finding also applies to the corresponding results for the free-stream velocity of $9 \mathrm{~m} / \mathrm{s}$ with no significant difference in the mean thickness of the UMZs as shown in figure $21(c, d)$.

Figure 22 shows the mean $\Delta_{u}$ obtained from the p.d.f. of $\Delta_{u}$ for each UMZ interface along 
(a)

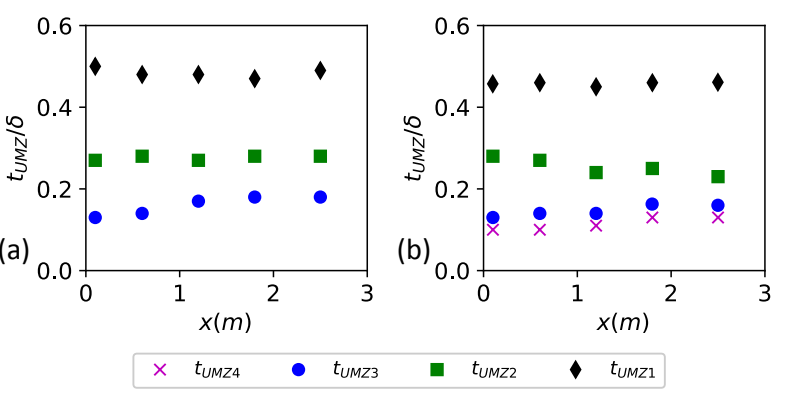

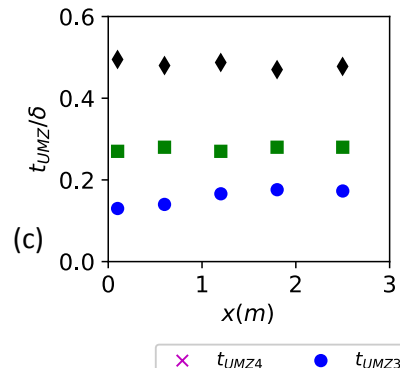

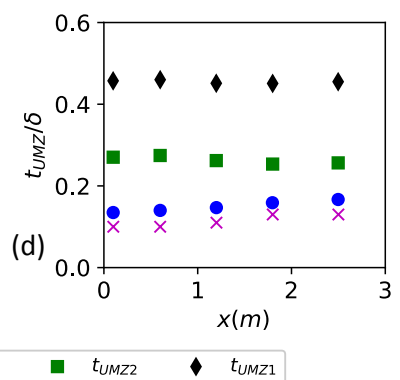

Figure 21: Variation of mean UMZ thickness at five streamwise locations $(x=0.1 \mathrm{~m}, 0.6 \mathrm{~m}$, $1.2 \mathrm{~m}, 1.8 \mathrm{~m}$ and $2.5 \mathrm{~m}$, where the value of $x$ represents the midpoint of each $2 \delta$ domain) along the APG domain for the velocity fields having 3 UMZs and 4 UMZs $(a, b)$ free-stream velocity of $5 \mathrm{~m} / \mathrm{s},(c, d)$ free-stream velocity of $9 \mathrm{~m} / \mathrm{s}$
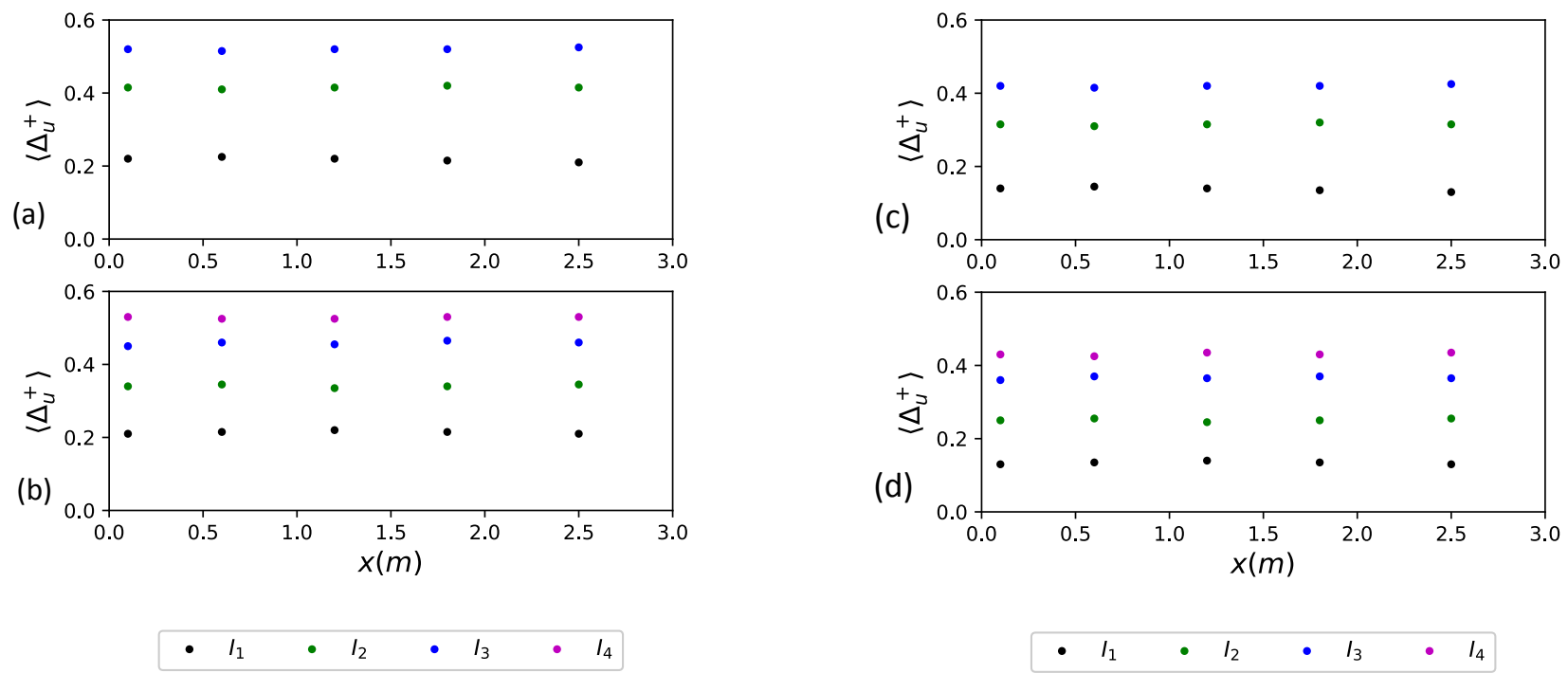

Figure 22: Variation of mean jump in streamwise velocity across each UMZ interface in the velocity fields having $3 \mathrm{UMZs}$ and $4 \mathrm{UMZs}$ at five streamwise locations along the APG domain for $(a, b)$ free-stream velocity of $5 \mathrm{~m} / \mathrm{s}$ and $(c, d)$ free-stream velocity of $9 \mathrm{~m} / \mathrm{s}$.

the APG domain. It is evident from these results that the observation found from the analysis of the p.d.f. of $\Delta_{u}$ at a single location, i.e. $x=0.6 \mathrm{~m}$, is consistent throughout the APG domain for both instantansous velocity fields having 3 and 4 UMZs. The average magnitudes of $\Delta_{u}$ across each of the UMZ interfaces remains relatively constant throughout the entire APG domain indicating that $\Delta_{u}$ is not influenced by increasing $R e_{\theta}$ nor $\beta$. This result also applies to the free-stream velocity of $9 \mathrm{~m} / \mathrm{s}$ case as evidenced in figure $22(c, d)$.

Figure 23 shows the variation of mean $\delta_{w}$ along the APG domain in inner and outer scalings. Under inner scaling shown in figure $23(a)$, a slight increase in $\delta_{w}$ is observed in the streamwise flow direction for both $N_{u m z}=3$ and 4 . In contrast when the results are shown in terms of outer scaling as shown in figure $23(b)$, a decreasing trend is observed. These observed variations in $\delta_{w}$ depending up the normalisation in terms of inner and outer scalings reflect the effect of the variation of the scaling parameters $u_{\tau}, \delta$ in an APG, since $u_{\tau}$ decreases faster compared to a 
(a)
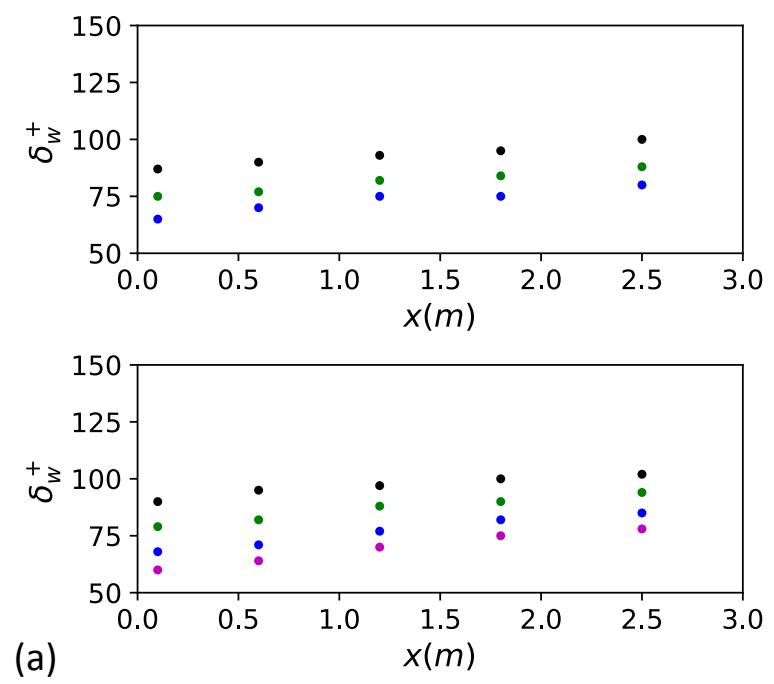

(b)
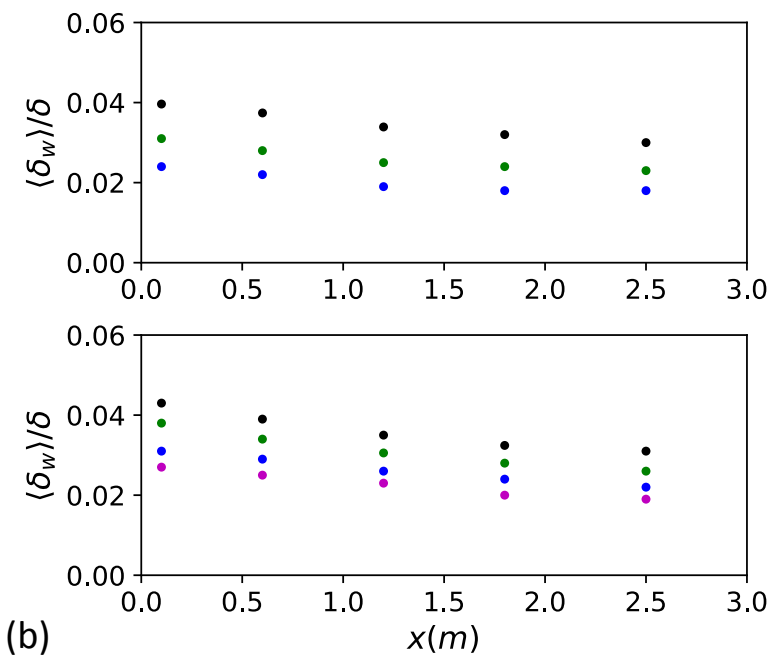

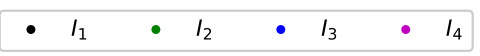
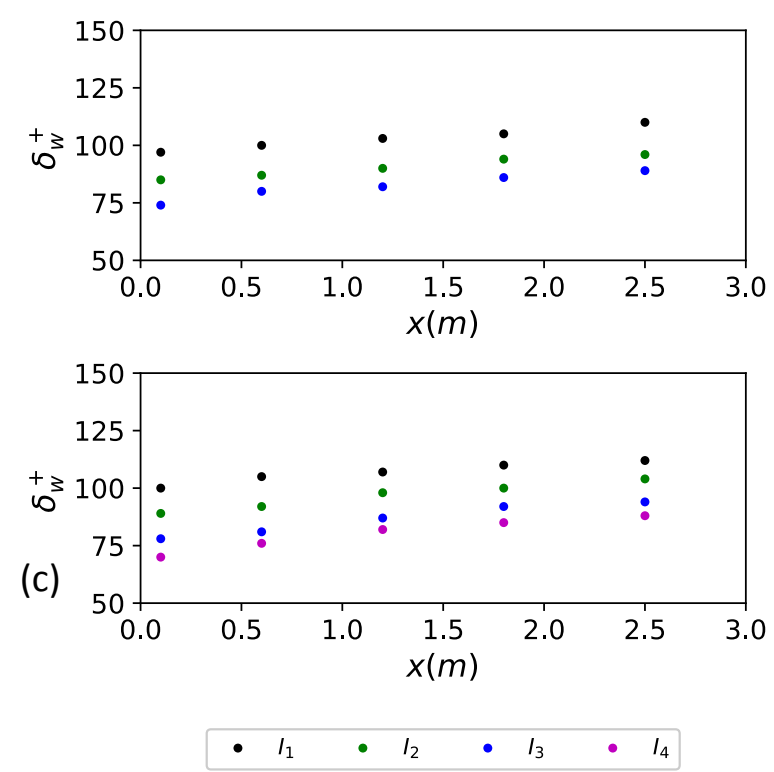
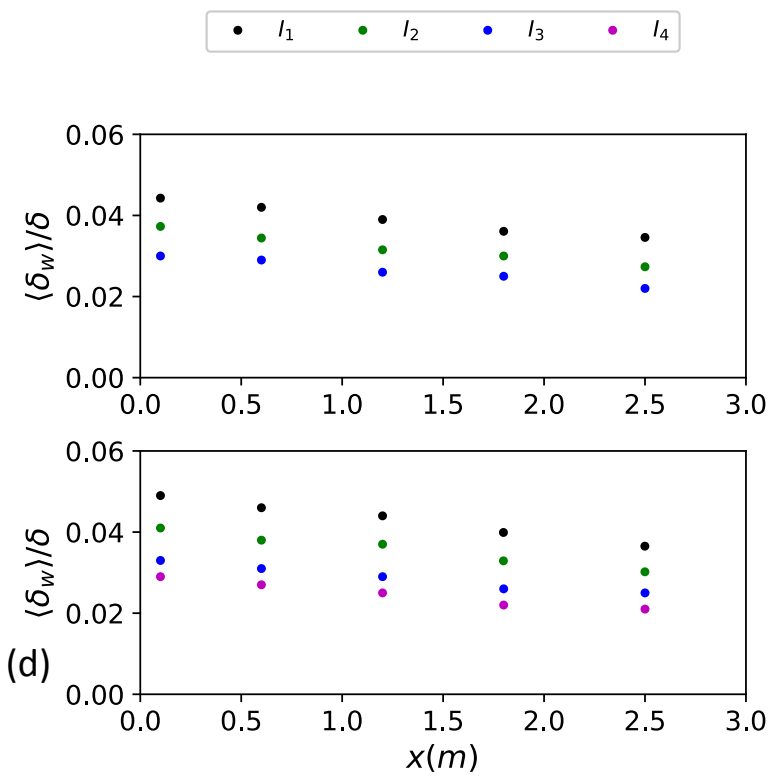

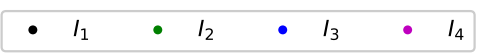

Figure 23: Variation of mean width of the UMZ interface along the APG domain for the velocity fields having 3 and 4 UMZs in the inner and the outer scaling, $(a, b)$ free-stream velocity of 5 $\mathrm{m} / \mathrm{s},(c, d)$ free-stream velocity of $9 \mathrm{~m} / \mathrm{s}$.

ZPG and $\delta$ increases faster compared to a ZPG along the APG domain. The same behaviour is observed for the free-stream velocity of $9 \mathrm{~m} / \mathrm{s}$ case as shown in figure $23(c, d)$.

\subsection{Influence of $N_{u m z}$ on turbulence statistics}

From the results presented in the previous sections, it is clear that number of UMZs present in the instantaneous field is an important factor that influences the characteristics of UMZs and UMZ interfaces. This influence may naturally contribute to the statistics of the APG-TBL. Hence in this section, the influence of $N_{u m z}$ on the streamwise Reynolds stress, Reynolds shear 

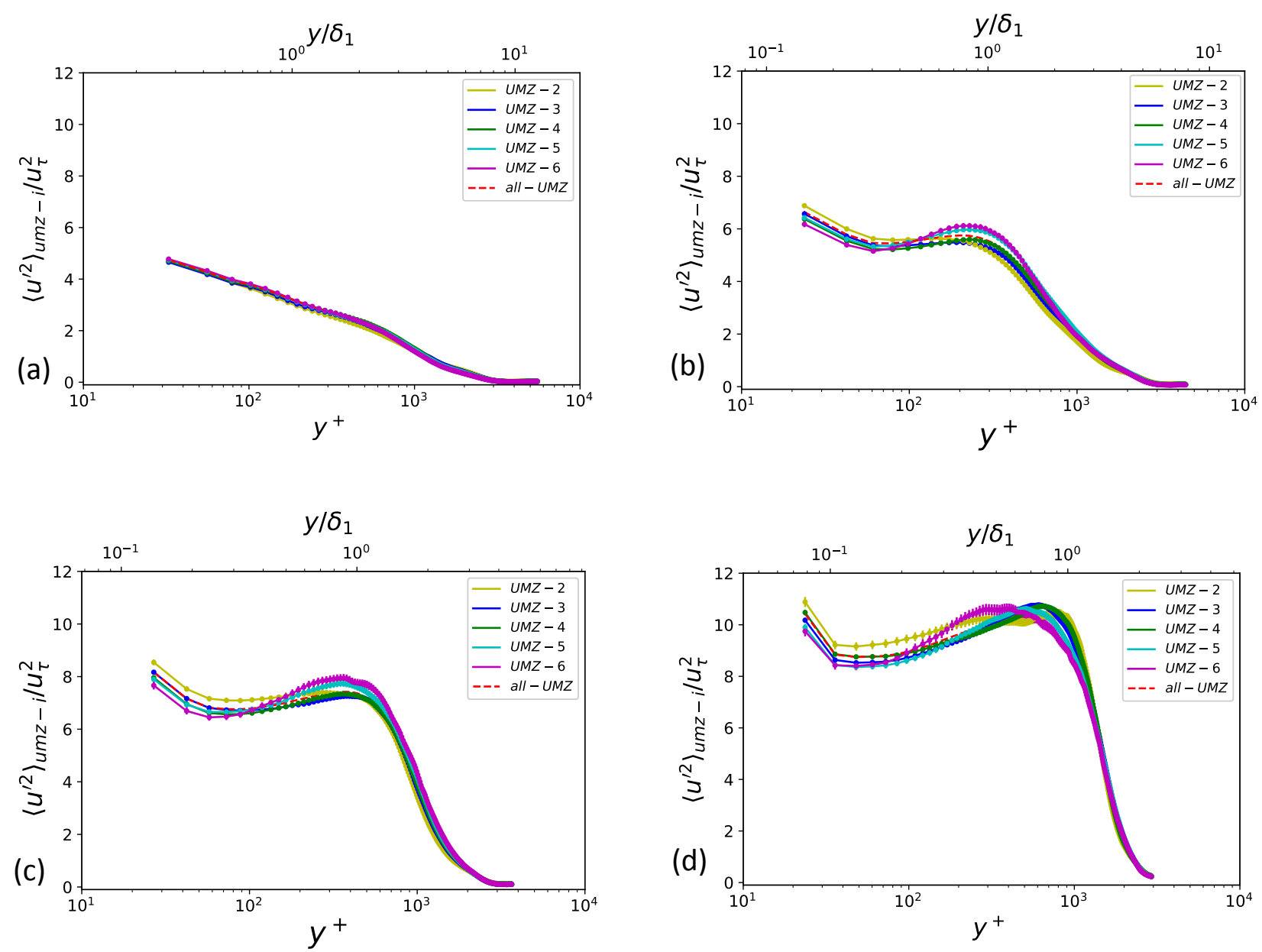

Figure 24: Comparison of streamwise Reynolds stress profiles between the velocity fields having 2, 3, 4, 5 and 6 UMZs along the APG domain for free-stream velocity of $5 \mathrm{~m} / \mathrm{s}(a)$ at $x=0.1$ $m, R e_{\theta}=5,495, \beta=0.45 ;(b)$ at $x=0.6 \mathrm{~m}, \operatorname{Re}_{\theta}=6,475, \beta=1.79 ;(c)$ at $x=1.5 \mathrm{~m}, \operatorname{Re}_{\theta}=$ $10,228, \beta=2.3 ;(d)$ at $x=2.9 m, R_{\theta}=11,942, \beta=3.79$. Error bars represent the uncertainty.

stress and turbulence production is examined by comparing the distributions of the quantities across the APG-TBL using the conditional statistics generated fro the sub-sets of the 30,000 samples that have $N_{u m z}=2,3,4,5$ and 6 within the $2 \delta$ streamwise extent used to determine the UMZs.

Figure 24 shows the variation of streamwise Reynolds stress profiles as a function of $N_{u m z}$ averaged over a streamwise domain extent of $2 \delta$ at four streamwise locations corresponding to $x=0.1 \mathrm{~m}, 0.6 \mathrm{~m}, 1.5 \mathrm{~m}$ and $2.9 \mathrm{~m}$ along the APG domain. At the start of the APG domain, $x$ $=0.1 \mathrm{~m}$, no difference in the magnitude of streamwise Reynolds stress is observed between the velocity fields having $N_{u m z}=2,3,4,5$ and 6 . As the flow evolves in the streamwise direction, a slight difference in the magnitude of streamwise Reynolds stress is observed in the inner and outer regions between the velocity fields having a low, i.e. $N_{u m z}=2$ and high, i.e. $N_{u m z}=$ 6. In the inner region, the magnitude of streamwise Reynolds stress is high for velocity fields having a low number of UMZs compared to velocity fields having a high number of UMZs, with the opposite applying in the outer region. These observations indicate that the number of UMZs present in the TBL influences the streamwise Reynolds stress in the flow. Similar 

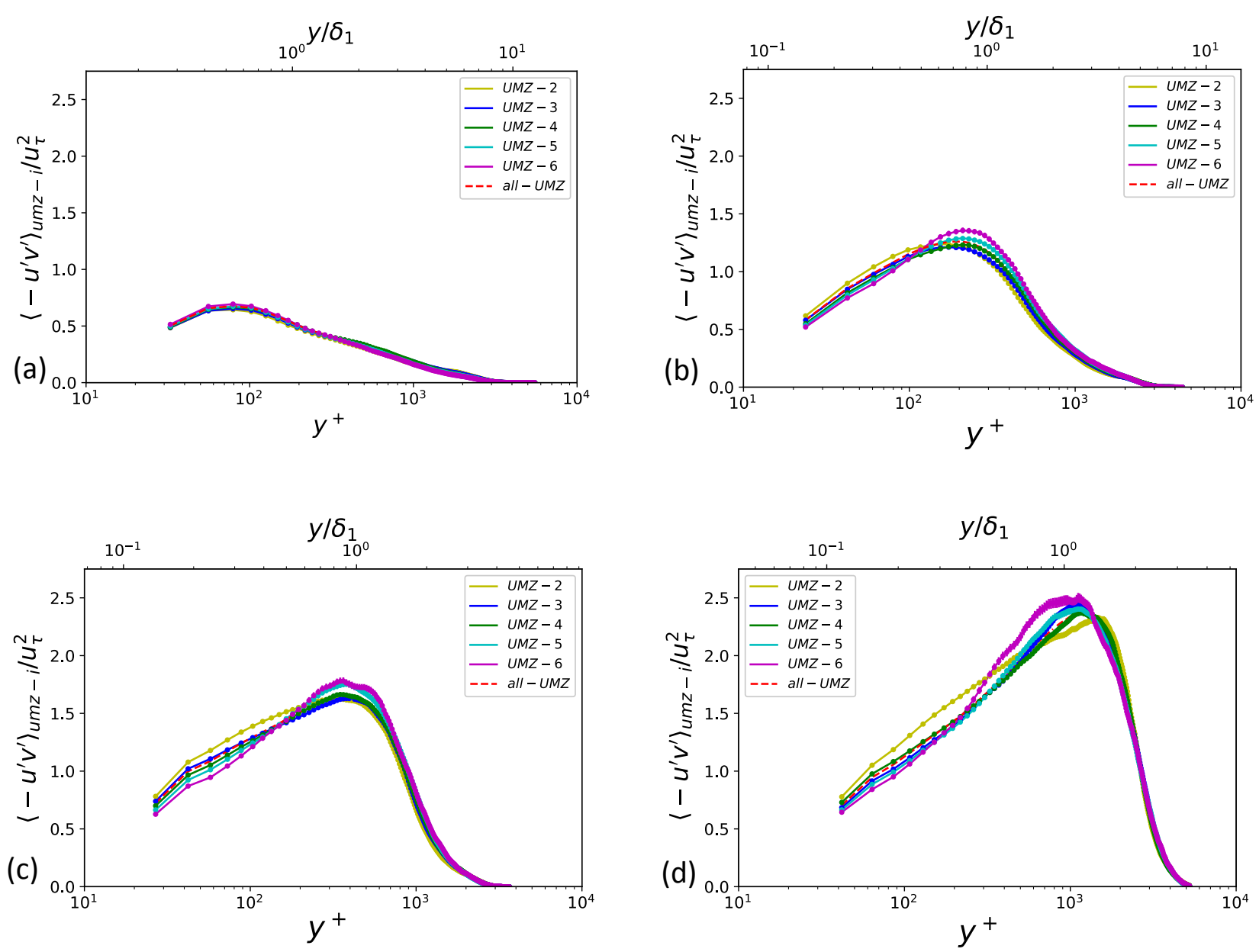

Figure 25: Comparison of Reynolds shear stress profiles between the velocity fields having 2, 3, 4, 5 and $6 \mathrm{UMZs}$ for a free-stream velocity of $5 \mathrm{~m} / \mathrm{s}(a)$ at $x=0.1 \mathrm{~m}, R e_{\theta}=5495, \beta=0.45$; (b) at $x=0.6 \mathrm{~m}, \operatorname{Re}_{\theta}=6,475, \beta=1.79 ;(c)$ at $x=1.5 \mathrm{~m}, \operatorname{Re}_{\theta}=10,228, \beta=2.3$; $(d)$ at $x=$ $2.9 \mathrm{~m}, \operatorname{Re}_{\theta}=11,942, \beta=3.79$. Error bars represent the uncertainty.

observations are found for the free-stream velocity of $9 \mathrm{~m} / \mathrm{s}$ case. It should be noted that the profiles corresponding to the velocity fields having 3 and 4 UMZs almost identically match the mean profile computed using all instantaneous velocity samples, i.e. all-UMZ, demonstrating that flow fields with $N_{u m z}=3$ or 4 are the most likely APG-TBL structure.

Figure 25 shows the variation of Reynolds shear stress profiles as a function of $N_{u m z}$ at four streamwise locations along the APG domain. Similar to the observation of streamwise Reynolds stress, at the start of the domain, i.e. $x=0.1 \mathrm{~m}$, no difference in the magnitude of Reynolds shear stress is observed between the velocity fields having $N_{u m z}=2,3,4,5$ and 6 . As the APG-TBL evolves in the streamwise direction a slight difference in the magnitude of Reynolds shear stress is found in the inner and outer regions between the velocity fields having a low and high number of UMZs. The magnitude of Reynolds shear stress is higher in the inner region for velocity fields having a low number of UMZs compared to the velocity fields having a high number of UMZs, and it is vice versa in the outer region. These observations clearly indicate that the number of UMZs present in the flow domain influence the shear stress distribution in the APG-TBL, with similar observation found for free-stream velocity of $9 \mathrm{~m} / \mathrm{s}$. 

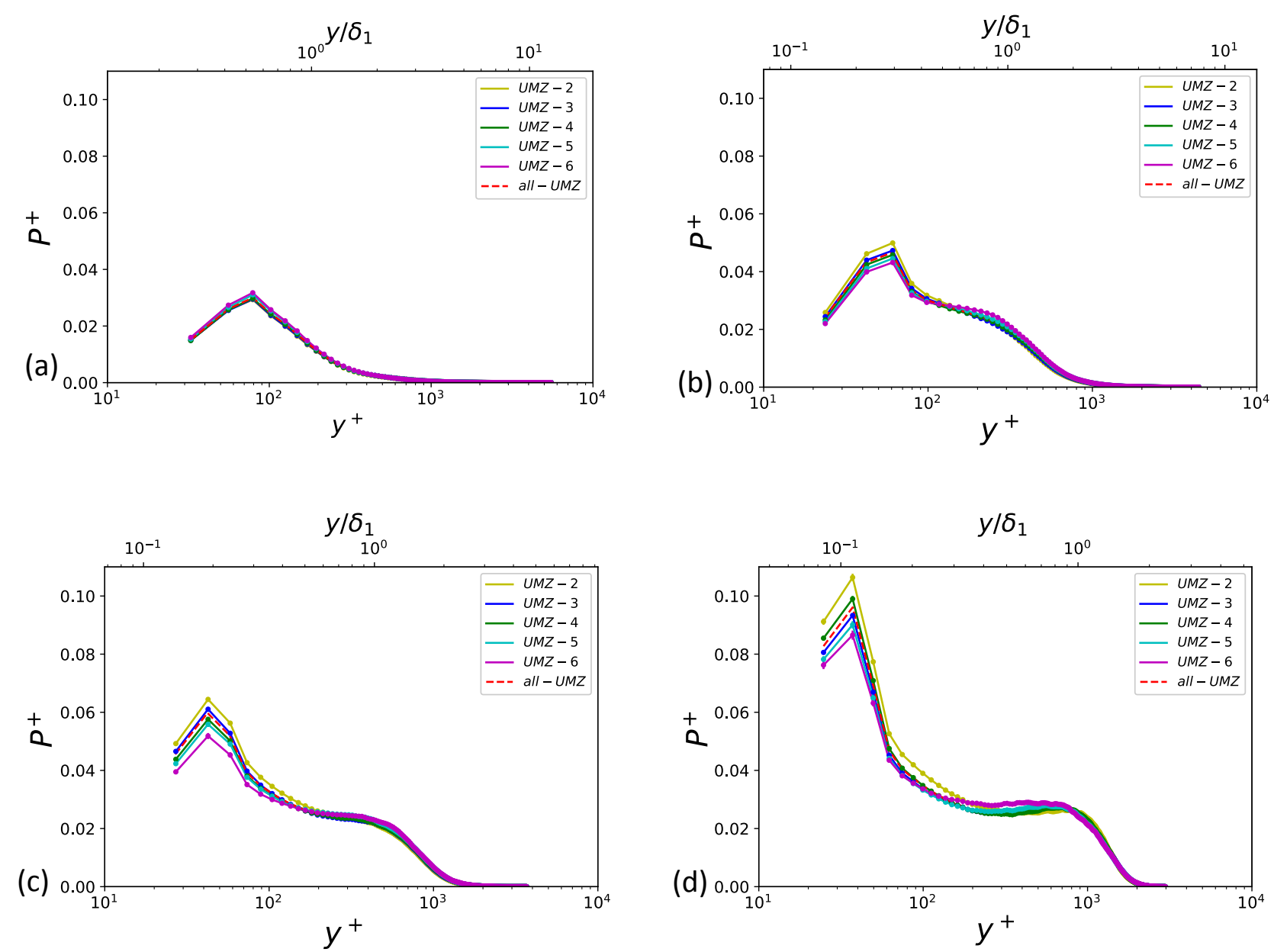

Figure 26: Comparison of turbulence production profiles between the velocity fields having 2, 3, 4, 5 and 6 UMZs for a free-stream velocity of $5 \mathrm{~m} / \mathrm{s}$ along the APG domain $(a)$ at $x=0.1$ $m, R e_{\theta}=5,495, \beta=0.45$; (b) at $x=0.6 \mathrm{~m}, \operatorname{Re}_{\theta}=6,475, \beta=1.79 ;(c)$ at $x=1.5 \mathrm{~m}, \operatorname{Re}_{\theta}=$ $10,228, \beta=2.3 ;(d)$ at $x=2.9 m, R e_{\theta}=11,942, \beta=3.79$. Error bars represent the uncertainty.

Figure 26 shows the comparison of turbulence production profiles as a function of $N_{u m z}=2$, $3,4,5$ and 6 at four streamwise locations along the APG domain. Analagously to the previous observations, no significant difference in the magnitude of production is observed between the velocity fields having $N_{u m z}=2,3,4,5$ and 6 at the start of the APG domain. As the APG-TBL evolves in the streamwise direction a slight difference in the magnitude of turbulence production is observed in the inner region between velocity fields having a low and high number of UMZs. The magnitude of production near the wall is slightly higher for velocity fields having a low number of UMZs compared to the velocity fields having a high number of UMZs. This difference in the turbulence production in the inner region can be associated with the difference observed in the Reynolds shear stress profiles in the inner region shown in figure 25. However, in the outer region, no difference in the magnitude of production is observed, indicating that the number of UMZs present in the flow only influences the turbulence production near the wall of an APG-TBL, with similar observation found for free-stream velocity of $9 \mathrm{~m} / \mathrm{s}$. 


\section{Concluding Remarks}

The existence of UMZs identified from the local peaks in the p.d.f. of the instantaneous streamwise velocity component along the APG domain using streamwise extents of $2 \delta$ reveals that UMZs are present in approximately $98 \%$ of the instantaneous velocity fields, confirming their presence throughout the APG-TBL. The constructing of the p.d.f. of the number of UMZs reveals that the most likely number of UMZs increases as the flow develops in the streamwise direction for both free-stream velocities of $5 \mathrm{~m} / \mathrm{s}$ and $9 \mathrm{~m} / \mathrm{s}$ investiagted in this study. It is observed that the most probably number of UMZs increases from 3 to 4 midway along the APG domain for both free-stream velocities at a $\beta$ value which is also the same for both free-stream velocities. Although the Reynolds number, $R e_{\theta}$ is different at that location for both free-stream velocities. The most likely cause for the increase in the most probable number of UMZs is most likely due to the enhanced boundary layer thickness growth in the APG environment, which sees an increasing $\beta$ in the streamwise flow direction. However, since no significant difference in the number of UMZs is observed between the two free-stream velocities, it can be summarised that there is most likely no Reynolds number effect on the most probable number of UMZs for the Reynolds number range examined in this study. Nevertheless, a clear relationship between $\beta$ and the most probable number of UMZs cannot be not established, since both $\beta$ and $R e_{\theta}$ vary along the APG domain and the effects of $\beta$ and $R e_{\theta}$ cannot be completely uncoupled.

The resulting number of UMZs identified in the 30,000 velocity field samples appear to influence the modal velocities and the geometrical properties of the UMZs. From the characteristics of UMZs based on the modal velocities in the same $2 \delta$ streamwise extent in the APG domain, it is evident that as $N_{u m z}$ increases, the modal velocity of each UMZ moves towards higher values to accommodate the new modal velocities, i.e. new UMZs, that are always found to develop near the wall. This development of new UMZs near the wall is in agreement with the recent findings of Laskari et al. [2018] and the attached eddy model of Townsend [1976], Head and Bandyopadhyay [1981], Perry and Chong [1982], which proposes that vortical structures scale with wall-normal distance and hence, have their origin at the wall.

The characterisation of UMZs based on the geometrical properties such as the thickness of UMZs, reveals that the thickness identified across the APG-TBL increases with increasing wall-normal distance, further supporting the hypothesis that further away from the wall, the length-scale associated with the UMZs increases. This pattern of increase in the thickness is consistent in the streamwise direction of the APG domain for both free-stream velocities and also agrees with the previous observations in the ZPG-TBL [Adrian, 1991, de Silva et al., 2016]. The influence of $N_{u m z}$ on the UMZ thicknesses is such that the thickness of each UMZ decreases with increasing $N_{u m z}$. This decrease in the UMZ thickness with increasing $N_{u m z}$ is further substantiated by the observed result of the wall-normal thickness of UMZs as a function of $N_{u m z}$, where no significant difference in the mean wall-normal height of the UMZ interface closest to the free-stream is observed between the velocity fields which have $N_{u m z}=2,3,4,5$ and 6. This indicates that as the $N_{u m z}$ increases, the UMZs are packed closer together resulting in a decreased (or compressed) thickness of each UMZ.

Additional properties investigated in this study are the jump in streamwise velocity $\left(\Delta_{u}\right)$ across the UMZ interface and the width of the UMZ interface $\left(\delta_{w}\right) . \Delta_{u}$ is found to decrease with increasing wall-normal distance. This observation of $\Delta_{u}$ agrees with the fact that closer to the wall the velocity gradient is higher in the APG-TBL. This variation of $\Delta_{u}$ in the wallnormal direction is consistent throughout the APG domain and their magnitude remains almost 
unchanged, indicating that $\Delta_{u}$ is not significantly influenced by increasing $\beta$ and $R e_{\theta}$ for both free-stream velocities. The width of the UMZ interface $\left(\delta_{w}\right)$ is found to increase with wallnormal distance, with a slight increase in $\delta_{w}$ observed along the APG domain under inner scaling, whereas it decreases under outer scaling for both free-stream velocities. This variation of $\delta_{w}$ in terms of either inner or outer scalings is due to the scaling parameters $u_{\tau}$ and $\delta$ used for the non-dimsnionalisation, as the $u_{\tau}$ decreases and $\delta$ increases along the APG domain. These observations are consistent with the results of de Silva et al. [2016], indicating that the UMZ and UMZ interface properties for the APG-TBL have similar behaviour to those of the ZPGTBL for the range of Reynolds number considered in this study. With the increasing $N_{u m z}, \Delta_{u}$ across each UMZ interface decreases, whereas $\delta_{w}$ increases. This decrease in $\Delta_{u}$ and increase in $\delta_{w}$ with increasing $N_{u m z}$ is due to the development of new UMZs in the near wall region causing the mean wall-normal location of the existing UMZs interface to move further away from the wall.

The effect of the characteristics of UMZs on Reynolds stresses and turbulence production were examined via conditional statistics. A slight difference in the magnitude of streamwise Reynolds stress and Reynolds shear stress is observed in both the inner and outer regions between the velocity fields having low number of UMZ, i.e. 2 UMZs and high number of UMZs, i.e. $6 \mathrm{UMZs}$, as the flow develops. It is found that the magnitude of the streamwise Reynolds stress and the Reynolds shear stress in the inner region are slightly higher for velocity fields that have a low number of UMZs compared to the velocity fields that have a high number of UMZs, with the opposite behaviour in the outer regions of the APG-TBL. This observation indicates that closer to the wall the turbulence activity is high for the velocity fields having a low number of UMZs compared to the velocity fields having a high number of UMZs, with the opposite behaviour in the outer region. These observations are also found to be in agreement with the recent findings of Laskari et al. [2018], who associated the increase in the turbulence activity in the log region of the velocity fields having a high number of UMZs with Q2 events, and the decrease in the turbulence activity in the log region of the velocity fields having a low number of UMZs with Q4 events. A slight difference in the magnitude of turbulence production is observed in the inner region between the velocity fields having a low or high number of UMZs, whereas no significant difference in the turbulence production is observed in the outer region. This indicates that the turbulence production near the wall is influenced by the $N_{u m z}$ present in the flow. Overall, these observations signify that the flow statistics are controlled by the number of UMZs present in the flow.

\section{Acknowledgments}

The authors are thankful to the EuHIT EC project (http://www.EUHIT.org) for the financial support which allowed the original experiment to take place. The C. Cuvier and J.M. Foucaut are thankful to CISIT, the Region Nord-Pas-de-Calais, the European community and the CNRS for the financial support and notably the one which has allowed making the test section fully transparent, permitting the experiment. The Monash team gratefully acknowledges the support of the Australian Research Council via a Discovery project grant. J. Soria gratefully acknowledges the support of an Australian Research Council Discovery Outstanding Researcher Award fellowship and Callum Atkinson the support of an Australian Research Council Discovery Early Career Research Award fellowship. 


\section{A Appendix}

\section{A.1 Identification of boundary layer parameters}

In the case of ZPG flow, the thickness of the boundary layer $(\delta)$ is defined as the wall-normal position at which the mean flow velocity $U(y)$ reaches $99 \%$ of free stream velocity. In the far-field location of APG flow, $\frac{\partial V}{\partial x}<0$, hence to have an irrotational flow in the far-field, i.e. zero spanwise vorticity, $\frac{\partial U}{\partial y}<0$. The mean velocity profile $(U)$ therefore has a turning point, and $\delta$ is defined as the wall-normal position of maximum velocity along the $U$ profile at which $d U(x, y) / d y \leqslant 0$ [Kitsios et al., 2016]. This formulation is used to identify the boundary layer thickness as it evolves downstream. Furthermore, the classical definition for displacement thickness $\left(\delta_{1}\right)$ and momentum thickness $\left(\delta_{2}\right)$ is not appropriate since the velocity profiles do not attain a constant value [Kitsios et al., 2017]. Hence, in this study, we use the definition of integral length scales $\left(\delta_{1}, \delta_{2}\right)$ and velocity length scales $\left(U_{e}, U_{\Omega}\right)$ from Spalart and Watmuff [1993], which are given by equations,

$$
\begin{gathered}
U_{e}(x)=U_{\Omega}(x, \infty) \\
U_{\Omega}(x, y)=-\int_{0}^{y}\left\langle\Omega_{z}\right\rangle(x, y) d y \\
\delta_{1}(x)=-\frac{1}{U_{e}} \int_{0}^{\infty} y \Omega_{z}(x, y) d y \\
\delta_{2}(x)=-\frac{2}{U_{e}^{2}} \int_{0}^{\infty} y U_{\Omega} \Omega_{z}(x, y) d y-\delta_{1}(x)
\end{gathered}
$$

where $\delta_{1}$ is the displacement thickness, $\delta_{2}$ is the momentum thickness, $U_{e}$ is the local free stream velocity and $\left\langle\Omega_{z}\right\rangle$ is the mean spanwise vorticity.

The Reynolds number commonly used to characterise the flow are the friction Reynolds number $\left(R e_{\tau}\right)$ and the momentum thickness based Reynolds number $\left(R e_{\theta}\right)$, which are given by equations,

$$
\begin{aligned}
& R e_{\tau}=\frac{u_{\tau} \delta}{\nu} \\
& R e_{\theta}=\frac{U_{e} \delta_{2}}{\nu}
\end{aligned}
$$

where $\delta$ is the thickness of boundary layer, $\delta_{2}$ is the momentum thickness, $\nu$ is the kinematic viscosity of the fluid, $u_{\tau}$ is the friction velocity and $U_{e}$ is the local free stream velocity. To compute $R e_{\tau}$, the friction velocity is identified using the relation

$$
u_{\tau}=\sqrt{\tau_{w} / \rho}
$$

where $\tau_{w}$ is the wall shear stress which is identified using the von Karman momentum integral equation. The wall shear stress is related to the momentum and displacement thickness by the von Karman momentum integral equation for two-dimensional developing flow given by, 


$$
\frac{\tau_{w}}{0.5 \rho U_{e}^{2}}=2 \frac{d \delta_{2}}{d x}+\frac{4 \delta_{2}+2 \delta_{1}}{U_{e}} \frac{d U_{e}}{d x}
$$

where $\rho$ is the density of the fluid [White, 2002]. In addition, the parameter which defines the strength of the APG flow relative to the wall shear stress is the non-dimensional pressure gradient parameter, $\beta$, [Clauser, 1954], which is defined as

$$
\beta=\frac{\delta_{1}}{\tau_{w}} \frac{d p}{d x}
$$

\section{A.2 Detection of TNTI}

The methodology followed by Chauhan et al. [2014] was used to identify the TNTI in the APG-TBL. The flow at the TNTI and over a small region above the TNTI is considered to be moving at a local free stream velocity. The local kinetic energy of fluctuation moving with a local free stream velocity over a $3 \times 32 \mathrm{D}$ velocity vector region is given by the equation,

$$
k_{1}=\frac{100}{9\left(U_{e}^{2}+V_{e}^{2}\right)} \sum_{m, n=-1}^{1}\left(\left(u_{m, n}-U_{e}\right)^{2}+\left(v_{m, n}-V_{e}\right)^{2}\right)
$$

where, $u$ and $v$ are the instantaneous velocity in the streamwise and wall-normal directions, $U_{e}$ and $V_{e}$ are the local free stream velocities, $m$ and $n$ represent the grid number. This normalised turbulent kinetic energy of fluctuation $\left(k_{1}\right)$ is nearly the same as the free stream turbulence intensity $\left(\overline{u^{\prime} u^{\prime}} / U_{e}^{2}\right.$, where $\overline{u^{\prime} u^{\prime}}$ is the variance of streamwise velocity fluctuations) above the interface and increases rapidly below the interface reaching a maximum value close to the wall [Chauhan et al., 2014]. This provides a basis to separate the turbulent and non-turbulent regions. For example, figure $27(a)$ shows the contour of $k_{1}$ for a single instantaneous field in a log scale as it varies from nearly zero in the non-turbulent region to a maximum value close to the wall. In the non-turbulent region, $k_{1} \approx 0$ and the value of $\ln k_{1}$ is negative in this region as shown in $27(a)$. The interface is detected by selecting an appropriate threshold limit of $k_{t h}$ as described by Chauhan et al. [2014].

Initially, a threshold value of $k_{t h}$ is selected that corresponds to the free-stream turbulence intensity. Regions with $k_{1}<k_{t h}$ are assigned a value of 1 by considering them as non-turbulent and the regions with $k_{1}>k_{t h}$ are assigned a value of 0 by considering them as turbulent. Once the regions are assigned with the binary values of zeros and ones, a contour line is generated in the single instantaneous frame. This line represents the TNTI, and the corresponding coordinates $\left(x_{i}, y_{i}\right)$ of the contour line define the interface. This process is repeated for the entire set of measured velocity fields, i.e. 30,000 velocity field samples. Then the mean $(\mu)$, and standard deviation $(\sigma)$ of the interface locations are obtained by ensemble average of interface coordinates across all 30,000 velocity field samples. The various values of $k_{t h}$ are checked at increments of 0.01 until $\mu \approx \delta$ to ensure that the interface coordinates are within $3 \sigma$ of the mean boundary layer thickness.

Following the above steps, a threshold value of $k_{t h}=0.02$ is selected. Figure $27(b)$ shows the interface determined using the $k_{t h}$ plotted over the contour of a single instantaneous streamwise velocity field. Figure 28 shows the p.d.f. of the instantaneous wall-normal location of the TNTI at different streamwise locations for the free-stream velocities of $5 \mathrm{~m} / \mathrm{s}$ and $9 \mathrm{~m} / \mathrm{s}$. The instantaneous wall-normal locations of TNTI lie above and below $\delta$ having the mean wall- 
(a)

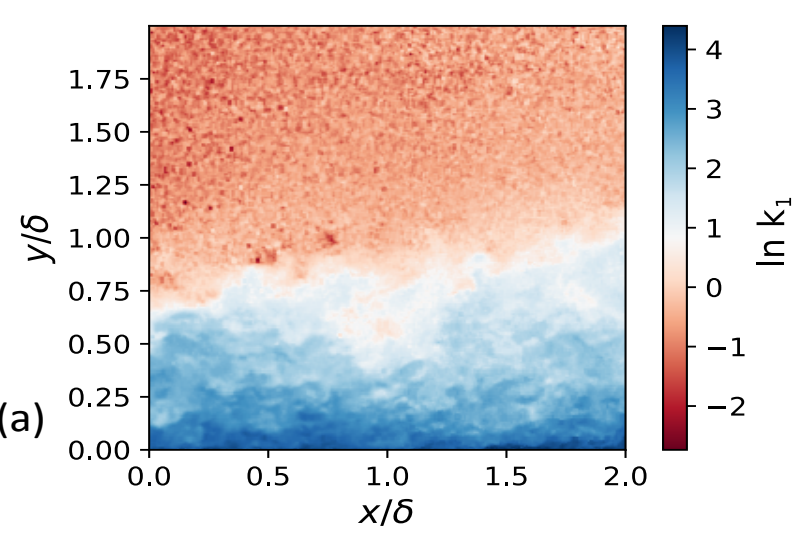

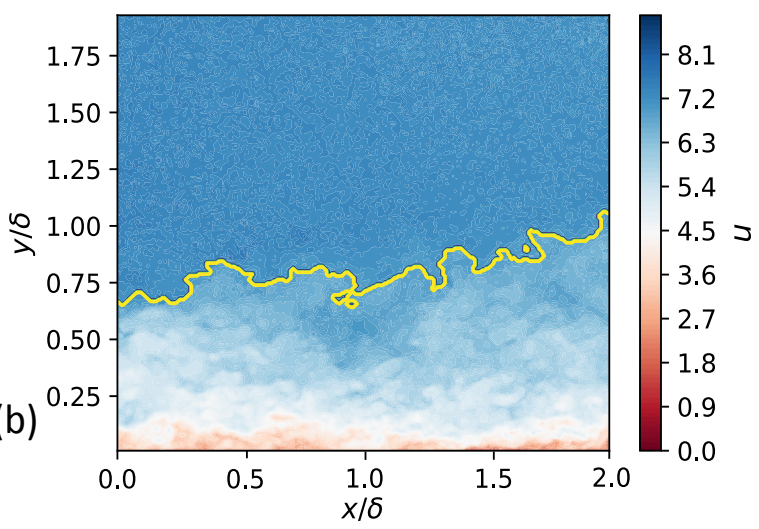

Figure 27: (a) Contour of $\ln k_{1}$ showing the turbulent (blue) and non-turbulent (red) region, (b) TNTI detected using $k_{t h}$ plotted over the contour of instantaneous streamwise velocity field covering a streamwise domain length of $2 \delta$.
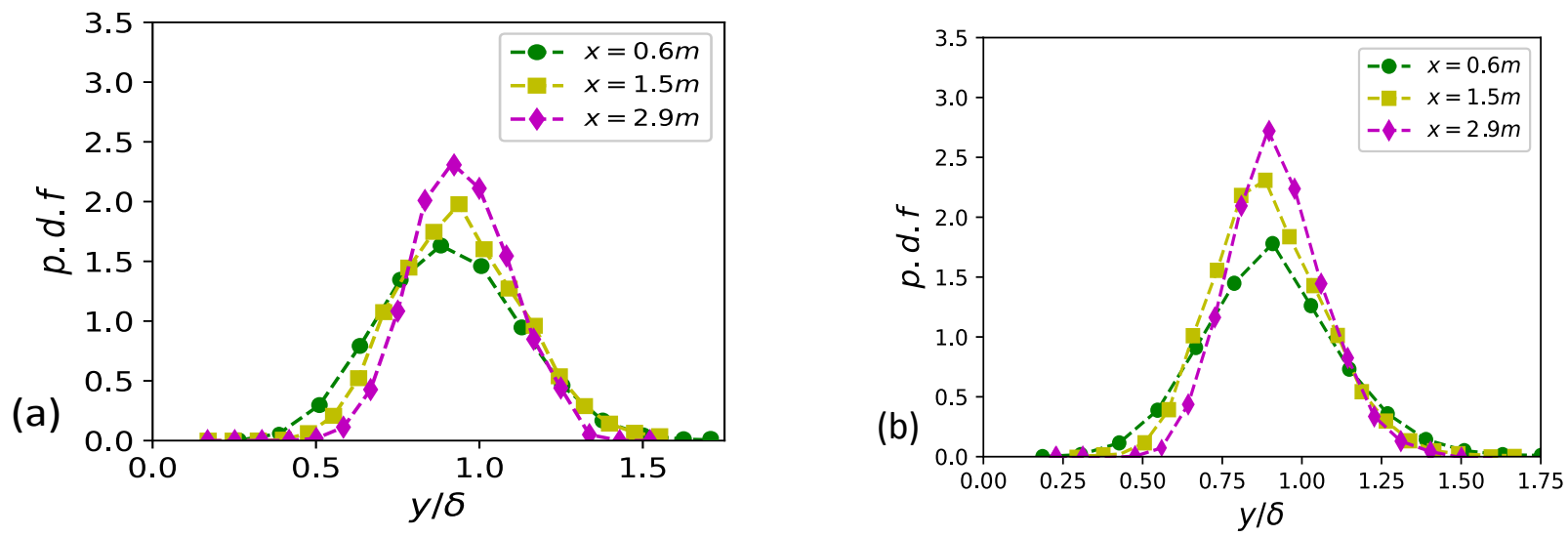

Figure 28: P.d.f. of instantaneous wall-normal location of TNTI at different streamwise locations, (a) $5 \mathrm{~m} / \mathrm{s}$, (b) $9 \mathrm{~m} / \mathrm{s}$.

normal location of TNTI nearly equal to $\delta$. This behaviour is consistent throughout the APG domain for both free-stream velocities.

\section{References}

M. S. Acarlar and C. R. Smith. A study of hairpin vortices in a laminar boundary layer, Part 2, Hairpin vortices generated by fluid injection. Journal of Fluid Mechanics, 175:43-83, 1987b.

R. J. Adrian. Particle-imaging techniques for experimental fluid mechanics. Ann. Rev. Fluid Mech., 23:261-304, 1991.

R. J. Adrian, C. D. Meinhart, and C. D. Tomkins. Vortex organization in the outer region of the turbulent boundary layer. Journal of Fluid Mechanics, 422:1-54, 2000. 
B. J. Balakumar and R. J. Adrian. Large and very large-scale motions in channel and boundarylayer flows. Phil. Trans. R. Soc., A 365:665-681, 2007.

R. F. Blackwelder and H. Eckelmann. Streamwise vortices associated with the bursting phenomenon. Journal of Fluid Mechanics, 94(03):577-594, 1979.

J. Carlier and M. Stanislas. Experimental study of eddy structures in a turbulent boundary layer using particle image velocimetry. Journal of Fluid Mechanics, 535(36):143-188, 2005.

K. Chauhan, J. Philip, C. M. de Silva, N. Hutchins, and I. Marusic. The turbulent/nonturbulent interface and entrainment in a boundary layer. Journal of Fluid Mechanics, 742:119-151, 2014.

K. T. Christensen and R. J. Adrian. Statistical evidence of hairpin vortex packets in wall turbulence. Journal of Fluid Mechanics, 431:433-443, 2001.

F. H. Clauser. Turbulent boundary layers in adverse pressure gradients. Journal of Aeronautical Sciences, 21:91-108, 1954.

C. Cuvier, S. Srinath, M. Stanislas, J. M. Foucaut, J. P. Laval, and C. J. Kähler. Extensive characterisation of a high Reynolds number decelerating boundary layer using advanced optical metrology. Journal of Turbulence, 18:929-972, 2017.

C. M. de Silva. Large-scale PIV measurements at high Reynolds numbers in wall-bounded turbulence. PhD thesis University of Melbourne, 2014.

C. M. de Silva, N. Hutchins, and I. Marusic. Uniform momentum zones in turbulent boundary layers. Journal of Fluid Mechanics, 786:309-331, 2016.

S. A. Dixit and O. N. Ramesh. Large-scale structures in turbulent and reverse-transitional sink flow boundary layers. Journal of Fluid Mechanics, 649:233-273, 2010.

G. E. Elsinga, D. J. Kuik, B. W. van Oudheusden, and F. Scarano. Investigationof the threedimensional coherent structures in a turbulent boundary layer with Tomographic-PIV. In 45th AIAA, Reno, Nevada, 2007.

B. Ganapathisubramani, E. K. Longmire, and I. Marusic. Characteristics of vortex packets in turbulent boundary layers. Journal of Fluid Mechanics, 478:35-46, 2003.

B. Ganapathisubramani, E. K. Longmire, and I. Marusic. Dual-plane PIV technique to determine the complete velocity gradient tensor in a turbulent boundary layer. Journal of Fluid Mechanics, 39:222-231, 2005.

M. R. Head and P. Bandyopadhyay. New aspects of turbulent boundary-layer structure. Journal of Fluid Mechanics, 107:297-338, 1981.

D. K. Heist, T. J. Hanratty, and Y. Na. Observations of the formation of streamwise vortices by rotation of arch vortices. Physics of Fluids, 12:2965-2975, 2000.

N. Hutchins, W. T. Hambleton, and I. Marusic. Inclined cross-stream stereo particle image velocimetry measurements in turbulent boundary layers. Journal of Fluid Mechanics, 541: 21-54, 2005. 
K. C. Kim and R. J. Adrian. Turbulence statistics in fully developed channel flow at low reynolds number. Journal of Fluid Mechanics, 177:133-166, 1987.

K. C. Kim and R. J. Adrian. Very large-scale motion in the outer layer. Physics of Fluids, 11: 417-422, 1999.

V. Kitsios, C. Atkinson, J. Sillero, G. Borrell, A. Gungor, J. Jiménez, and J. Soria. Direct numerical simulation of a self-similar adverse pressure gradient turbulent boundary layer. International Journal of Heat and Fluid Flow, 16:129-136, 2016.

V. Kitsios, A. Sekimoto, C. Atkinson, J. A. Sillero, G. Borrell, A. G. Gungor, J. Jimenez, and J. Soria. Direct numerical simulation of a self-similar adverse pressure gradient turbulent boundary layer at the verge of separation. Journal of Fluid Mechanics, 829:392-419, 2017.

S. J. Kline, W. C. Reynolds, F. A. Schraub, and P. W. Runstadler. The structure of turbulent boundary layers. Journal of Fluid Mechanics, 30:741-773, 1967.

A. Laskari, R. de Kat, R. J. Hearst, and B. Ganapathisubramani. Time evolution of uniform momentum zones in a turbulent boundary layer. Journal of Fluid Mechanics, 842:554-590, 2018.

J. Lee, J. H. Lee, J. I. Choi, and H. J. Sung. Spatial organization of large- and very-largescale motions in a turbulent channel flow. Journal of Fluid Mechanics, 749:818-840, 2014.

J. H. Lee and H. J. Sung. Very-large-scale motions in a turbulent boundary layer. Journal of Fluid Mechanics, 673:80-120, 2011.

I. Marusic. On the role of large-scale structures in wall turbulence. Journal of Fluid Mechanics, 13:735, 2001.

C. D. Meinhart and R. J. Adrian. On the existence of uniform momentum zones in a turbulent boundary layer. Physics of Fluids, 7:694, 1995.

Y. Nagano, T. Tsuji, , and T. Houra. Structure of turbulent boundary layer subjected to adverse pressure gradient. Int. J. Heat Fluid Flow, 19:563-572, 1998.

G. R. Offen and S. J. Kline. Combined dye-streak and hydrogen-bubble visual observations of a turbulent boundary layer. Journal of Fluid Mechanics, 62(02):223-239, 1974.

G. R. Offen and S. J. Kline. A proposed model of the bursting process in turbulent boundary layers. Journal of Fluid Mechanics, 70(02):209-228, 1975.

A. E. Perry and M. Chong. On the mechanism of wall turbulence. Journal of Fluid Mechanics, 119(173):106-121, 1982.

J. Philip and I. Marusic. Large-scale eddies and their role in entrainment in turbulent jets and wakes. Physics of Fluids, 24:055108, 2012.

J. Philip, C. Meneveau, C. M. de Silva, and I. Marusic. Multiscale analysis of fluxes at the turbulent/non-turbulent interface in high Reynolds number boundary layers. Physics of Fluids, 26(1):015105, 2014. 
M. Skote, D. S. Henningson, and R. A. W. M. Henkes. Direct numerical simulation of self-similar turbulent boundary layers in adverse pressure gradients. Flow, Turbulence and Combustion, 60:47-85, 1998 .

J. Soria. An investigation of the near wake of a circular cylinder using a video-based digital cross-correlation particle image velocimetry technique. Experimental Thermal and Fluid Science, 12:221-233, 1996.

J. Soria. Multigrid approach to cross-correlation digital PIV and HPIV analysis. In 13th Australasian Fluid Mechanics Conference, pages 381-384, Monash University, Melbourne, 1998.

P. Spalart and J. Watmuff. Experimental and numerical study of a turbulent boundary layer with pressure gradients. Journal of Fluid Mechanics, 249:337-371, 1993.

K. R. Sreenivasan and R. A. Antonia. Properties of wall shear stress fluctuations in a turbulent duct flow. ASME, Trans., 44:389-395, 1977.

K. R. Sreenivasan and C. Meneveau. The fractal facets of turbulence. Journal of Fluid Mechanics, 173(1):357-386, 1986.

T. Theodersen. Mechanism of turbulence. In Proceedings of the Mid-western Conference on Fluid Mechanics, 23(1):601-639, 1952.

C. D. Tomkins and R. J. Adrian. Spanwise structure and scale growth in turbulent boundary layers. Journal of Fluid Mechanics, 490(1):37-74, 2003.

A. A. Townsend. The structure of Turbulent Shear Flow. 2nd ed., Cambridge University Press, 1976.

J. M. Wallace, H. Eckelmann, and R. S. Brodkey. The wall region of turbulent shear flow. Journal of Fluid Mechanics, 54:39-48, 1972.

J. Westerweel, C. Fukushima, J. M. Pedersen, and J. C. R. Hunt. Momentum and scalar transport at the turbulent/non-turbulent interface of a jet. Phys. Rev. Lett., 95:174501, 2005.

F. M. White. Viscous flow. McGraw Hill, New York, 2002.

C. Willert and M. Gharib. Digital particle image velocimetry. Experiments in Fluids, 10: 181-193, 1991. 